OPEN ACCESS

\section{Abelian hidden sectors at a $\mathrm{GeV}$}

To cite this article: David E. Morrissey et al JHEP07(2009)050

View the article online for updates and enhancements.

\section{Related content}

Local models of gauge mediated supersymmetry breaking in string theory Iñaki García-Etxebarria, Fouad Saad and Angel M. Uranga

Common gauge origin of discrete symmetries in observable sector and hidden sector

Taeil Hur, Hye-Sung Lee and Christoph Luhn

- Semi-direct gauge mediation Nathan Seiberg, Tomer Volansky and Brian Wecht

\section{Recent citations}

- Dark matter in very supersymmetric dark
sectors
Avital Dery et al
- A portalino to the dark sector
Martin Schmaltz and Neal Weiner
- Liaht dark matter from leptogenesis
Adam Falkowski et al




\title{
Abelian hidden sectors at a GeV
}

\author{
David E. Morrissey, ${ }^{a}$ David Poland ${ }^{a}$ and Kathryn M. Zurek ${ }^{b, c}$ \\ a Jefferson Physical Laboratory, Harvard University, \\ Cambridge, Massachusetts 02138, U.S.A. \\ ${ }^{b}$ Particle Astrophysics Center, Fermi National Accelerator Laboratory, \\ Batavia, Illinois 60510, U.S.A. \\ ${ }^{c}$ Department of Physics, University of Michigan, \\ Ann Arbor, Michigan 48109, U.S.A. \\ E-mail: dmorriss@physics.harvard.edu, dpoland@physics.harvard.edu, \\ kzurek@fnal.gov
}

ABSTRACT: We discuss mechanisms for naturally generating GeV-scale hidden sectors in the context of weak-scale supersymmetry. Such low mass scales can arise when hidden sectors are more weakly coupled to supersymmetry breaking than the visible sector, as happens when supersymmetry breaking is communicated to the visible sector by gauge interactions under which the hidden sector is uncharged, or if the hidden sector is sequestered from gravity-mediated supersymmetry breaking. We study these mechanisms in detail in the context of gauge and gaugino mediation, and present specific models of Abelian GeV-scale hidden sectors. In particular, we discuss kinetic mixing of a $\mathrm{U}(1)_{x}$ gauge force with hypercharge, singlets or bi-fundamentals which couple to both sectors, and additional loop effects. Finally, we investigate the possible relevance of such sectors for dark matter phenomenology, as well as for low- and high-energy collider searches.

KeYwords: Beyond Standard Model, Cosmology of Theories beyond the SM, Supersymmetric gauge theory, Supersymmetry Breaking

ARXIV EPRINT: 0904.2567 


\section{Contents}

1 Introduction 2

2 Supersymmetry breaking and the hidden sector 3

2.1 Supergravity effects 4

2.2 Hypercharge kinetic mixing and D terms 5

$\begin{array}{lll}2.3 & \text { Little gauge mediation } & 7\end{array}$

2.4 Little gaugino mediation 8

2.5 MSSM renormalization group effects 9

2.6 Additional mediator fields 9

3 Models 11

3.1 Minimal $\mu^{\prime}$ model 12

3.1.1 $\mathbf{m}_{\mathbf{3} / \mathbf{2}} \ll \mathbf{m}_{\text {hid }} \quad 13$

$\begin{array}{lll}3.1 .2 & \mathbf{m}_{\mathbf{3} / \mathbf{2}} \gtrsim \mathbf{m}_{\text {hid }} & 15\end{array}$

$\begin{array}{lll}3.2 & \text { Hidden sector NMSSM } & 17\end{array}$

$\begin{array}{lll}3.2 .1 & \mathbf{m}_{\mathbf{3} / \mathbf{2}} \ll \mathbf{m}_{\text {hid }} & 18\end{array}$

$\begin{array}{lll}3.2 .2 & \mathbf{m}_{\mathbf{3} / \mathbf{2}} \gtrsim \mathbf{m}_{\text {hid }} & 20\end{array}$

3.3 Singlet-mediated SUSY breaking 22

$3.3 .1 \mathbf{m}_{\mathbf{3} / \mathbf{2}} \ll \mathbf{m}_{\text {hid }} \quad 24$

$\begin{array}{ll}3.3 .2 & \mathbf{m}_{\mathbf{3} / \mathbf{2}} \gtrsim \mathbf{m}_{\text {hid }}\end{array}$

3.4 Multi-mediator models 25

4 Signatures in dark matter searches and colliders $\quad 26$

4.1 Dark matter direct detection and DAMA 26

$\begin{array}{lll}\text { 4.1.1 Elastic dark matter } & 27\end{array}$

$\begin{array}{lll}\text { 4.1.2 Inelastic dark matter } & 28\end{array}$

4.2 Applications to PAMELA and ATIC 30

$\begin{array}{ll}4.3 \text { Collider phenomenology } & 30\end{array}$

$\begin{array}{lll}\text { 4.3.1 High-energy hadron colliders } & 31\end{array}$

$\begin{array}{lll}\text { 4.3.2 } & \text { Lower-energy } e^{+} e^{-} \text {colliders } & 32\end{array}$

5 Conclusions $\quad 33$

$\begin{array}{ll}\text { A Kinetic mixing mediation } & 34\end{array}$

B Renormalization group equations $\quad 36$

B.1 Minimal $\mu^{\prime}$ model $\quad 36$

$\begin{array}{lll}\text { B.2 Hidden sector NMSSM } & 36\end{array}$ 


\section{Introduction}

In the minimal supersymmetric extension of the standard model (MSSM), the scale of electroweak symmetry breaking is determined by and is on the order of the scale of soft supersymmetry breaking [1]. Similarly, the effective amount of supersymmetry breaking in other sectors of the theory can naturally induce gauge symmetry breaking at the corresponding mass scale. If the breaking of supersymmetry is communicated predominantly by gravitational interactions, the scale of supersymmetry breaking is typically very similar for all sectors of theory, even if they do not couple appreciably to one another [2,3]. However, if supersymmetry breaking is communicated by gauge interactions $[4,5]$ under which certain sectors of the theory are uncharged, a hierarchy among the scales of supersymmetry breaking can arise between the different sectors [6-14].

A simple and concrete example of the class of scenarios that we consider in the present work consists of the MSSM augmented by an additional hidden $\mathrm{U}(1)_{x}$ sector. Effects proportional to the scale of supersymmetry breaking can enter into the hidden sector in several ways. Supergravity interactions are always expected to be present, though their size depends on the gravitino mass $m_{3 / 2}$, and whether or not there is sequestering of generic $M_{\mathrm{Pl}}$-suppressed operators $[15,16]$.

The $\mathrm{U}(1)_{x}$ sector can also have a renormalizable coupling to the MSSM through kinetic mixing with hypercharge. Such a term can induce an effective Fayet-Illiopoulos term in the hidden sector when there is a $D$ term for hypercharge [11-13], which can cause the hidden gauge group to break. For natural values of the gauge kinetic mixing, the symmetry breaking scale is on the order of a $\mathrm{GeV}$. In addition, if supersymmetry breaking is communicated to the visible sector through the SM gauge interactions (e.g., assuming gauge or gaugino mediation), SUSY-breaking effects will be transferred in turn to parameters in the hidden sector at the messenger scale in the presence of kinetic mixing. Renormalization group effects from the visible-sector gauginos can also induce soft parameters in the hidden sector through the kinetic mixing term. These effects are naturally less than or on the order of the GeV scale.

If singlets are present in the hidden sector, they may also communicate supersymmetry breaking if they couple directly to the messenger sector or to the MSSM. With such singlets, no kinetic mixing is necessary to communicate SUSY breaking to the hidden sector. In this case, the SUSY-breaking scale in the hidden sector can again be around a GeV.

All these effects combine to suggest that hidden sectors may be found around the $\mathrm{GeV}$ scale. Such new sectors are consistent with current experimental bounds provided they are sufficiently hidden, which in the case of gauge kinetic mixing corresponds to $\epsilon \lesssim 10^{-2}[17]$. The detailed phenomenology of these hidden sectors and their cosmological viability, however, depends strongly on the relative size of the gravitino mass compared with the mass of the lightest hidden-sector particle (LHP). If the gravitino is much lighter and the hidden sector respects R-parity, the lightest R-odd particle will typically be long lived and give rise to problematic decays after nucleosynthesis or be overabundant. If the gravitino is heavier, then the lightest R-odd particle will be stable and one still needs to ensure that it has an efficient annihilation channel. We will show that this is indeed possible in the context of simple hidden-sector models. 
New hidden sectors at a GeV may help to explain some of the recent, but surprising, hints for dark matter (DM). The positron and electron excesses seen by PAMELA [18], ATIC [19], and PPB-BETS [20], as well as the WMAP haze [21-23], can arise from dark matter annihilation, but seem to require an enhanced annihilation rate today relative to the value yielding the correct thermal relic density. This feature can arise from a low-velocity Sommerfeld enhancement of the annihilation in our galaxy, which for electroweak-scale dark matter requires an attractive force with a mediator lighter than a few $\mathrm{GeV}$ [8, 24]. New GeV-mass states are also suggested by the observation of an annual modulation signal at DAMA [25]. This can potentially be explained by the elastic scattering of GeV-mass dark matter states [26-31], or by heavier inelastic dark matter whose inelastic splittings and scattering cross-section emerge naturally through its coupling to new GeV-mass states [12, 32-35]. A GeV sector may also help to account for the $511 \mathrm{keV}$ line observed by the INTEGRAL [36] experiment.

Some of the mechanisms to generate light sectors which we discuss here have already been used to construct natural supersymmetric $\mathrm{MeV} \mathrm{U}(1)_{x}$ dark sectors, motivated by the possibility of $\mathrm{MeV}$-mass dark matter [6, 17, 37-39]. The difference between the $\mathrm{GeV}$ sectors which are the focus of this study and $\mathrm{MeV}$ sectors relevant there is the strength of the coupling of the hidden sector to SUSY breaking. In GeV-scale supersymmetric sectors, typical couplings to the MSSM and SUSY breaking are of size $10^{-3} \sim \mathrm{GeV} / \mathrm{TeV}$; by contrast, $\mathrm{MeV}$ sectors must have weaker couplings of size $10^{-6} \sim \mathrm{MeV} / \mathrm{TeV}$. However, some of the mechanisms we discuss in the present work can be applied to $\mathrm{MeV}$ sectors as well. Our study also has overlap with refs. $[13,14]$ that appeared while the present work was in preparation. Where there is overlap, we confirm their results.

The outline of this paper is as follows. In section 2 we investigate various ways to mediate supersymmetry breaking to $\mathrm{GeV}$-scale hidden sectors. Using these results, we construct several concrete Abelian hidden-sector models in section 3. In section 4 we investigate some applications of these models to explain recent hints for dark matter, and we discuss briefly their collider signatures. Finally, section 5 is reserved for our conclusions.

\section{Supersymmetry breaking and the hidden sector}

We begin with an overview of the different ways that supersymmetry breaking can be mediated to a hidden sector containing a $\mathrm{U}(1)_{x}$ gauge symmetry. Throughout this discussion, we assume that the MSSM feels supersymmetry breaking primarily through standard model $\mathrm{SU}(3)_{c} \times \mathrm{SU}(2)_{L} \times \mathrm{U}(1)_{Y}$ gauge interactions in the form of gauge mediation $[4,5]$ or gaugino mediation $[40,41]$. In the context of gauge mediation, we assume further that the messengers of supersymmetry breaking to the MSSM are not charged under the hidden-sector gauge group, and that there is no significant direct coupling of the hidden sector to the source of supersymmetry breaking. Within gaugino mediation, we assume that the hiddensector fields (including the Abelian gauge fields) are sequestered away from supersymmetry breaking. In both the gauge and gaugino mediation frameworks, supersymmetry breaking can be communicated to the hidden sector through a combination of supergravity interactions, kinetic mixing of the hidden $\mathrm{U}(1)_{x}$ gauge group with hypercharge, bi-fundamentals 
charged under both the hidden and visible gauge groups, and singlets that couple to both sectors. We describe each of these possible contributions below.

\subsection{Supergravity effects}

A strong motivation for gauge or gaugino mediation of supersymmetry breaking, relative to generic gravity mediation, is that these gauge-based mediation mechanisms provide an explanation for the absence of strong flavor mixing induced by $\mathrm{TeV}$-scale soft masses. For this to be effective, the MSSM soft terms induced by gauge or gaugino mediation must strongly dominate over those from supergravity couplings. Even so, residual supergravity effects can still potentially provide an important contribution to the suppressed soft terms in a hidden $\mathrm{U}(1)_{x}$ sector, and those that break $\mathrm{U}(1)_{R}$ symmetry in particular.

The typical size of supergravity effects in both the visible and hidden sectors can be described in terms of the gravitino mass $m_{3 / 2}[2,3]$,

$$
m_{3 / 2}=\frac{F}{\sqrt{3} M_{\mathrm{Pl}}}
$$

where $F$ parametrizes the underlying supersymmetry breaking. Generic $M_{\mathrm{Pl}^{-}}$suppressed operators connecting the source of supersymmetry breaking to other fields then give contributions to the soft parameters in all sectors of the theory on the order of the gravitino mass $m_{3 / 2}$. This leads to a quite general statement - in the absence of any other physics connecting the visible and hidden sectors, mass scales in hidden sectors are generically comparable to the gravitino mass.

An exception to this statement occurs when the generic $M_{\mathrm{Pl}}$-suppressed operators mediating supersymmetry breaking are further suppressed through sequestering. Sequestering can arise either by localizing a hidden sector on a brane away from the source of supersymmetry breaking $[15,16]$ or through conformal running effects [42]. ${ }^{1}$ When sequestering occurs, supergravity effects will still mediate supersymmetry breaking to all sectors of the theory through anomaly mediation $[15,16]$, generating soft masses on the order of

$$
\Delta m_{1 / 2}^{\mathrm{AMSB}} \sim \frac{g^{2}}{(4 \pi)^{2}} m_{3 / 2},
$$

where $g$ represents a coupling of the corresponding field. Anomaly-mediated soft terms do not generate too much flavor mixing [47], but can induce unacceptable tachyonic slepton scalar masses [15].

In comparison to these supergravity-mediated soft terms, gauge-mediated soft terms are on the order of

$$
m_{\mathrm{soft}}^{\mathrm{vis}} \sim \frac{g^{2}}{(4 \pi)^{2}} \frac{F}{M} \sim \frac{g^{2}}{(4 \pi)^{2}}\left(\frac{M_{\mathrm{Pl}}}{M}\right) m_{3 / 2},
$$

where $M$ is the messenger mass scale. Thus, gravity-mediated soft masses in both the visible and hidden sectors on the order of a $\mathrm{GeV}$ can arise for messenger masses close

\footnotetext{
${ }^{1}$ For a pedagogical review of conformal sequestering, see [43]. In addition to allowing for anomaly mediation to dominate, conformal sequestering effects have also recently been proposed as a solution to the $\mu / B \mu$-problem in gauge and gaugino mediation [44-46].
} 
to $M \sim 10^{14} \mathrm{GeV}$. This is about as large as possible while still being consistent with constraints on new sources of flavor mixing (assuming no new flavor symmetries) [48, 49].

Within gaugino mediation, generic supergravity effects are suppressed by sequestering the source of supersymmetry breaking. The leading soft terms generated are the visiblesector gaugino masses, on the order of

$$
m_{\mathrm{soft}}^{\mathrm{vis}} \sim g^{2}\left(\frac{M_{c}}{\Lambda}\right) \frac{F}{M_{c}} \sim g^{2}\left(\frac{M_{c}}{\Lambda}\right) \frac{M_{\mathrm{Pl}}}{M_{c}} m_{3 / 2},
$$

where $M_{c}$ is the compactification scale (inverse of the compactification length) and $\Lambda$ is the higher-dimensional cutoff scale. The ratio $M_{c} / \Lambda$ is less than unity and can be as small as a loop factor.

Even with sequestering, residual supergravity effects in all sectors of the theory will arise from anomaly mediation. These will be at the GeV scale when $m_{3 / 2} \sim 100 \mathrm{GeV}$, corresponding to $M_{c} \sim g^{2}\left(M_{c} / \Lambda\right) M_{\mathrm{Pl}}$. Thus, in this case the cutoff scale must be close to the Planck scale. Note that this counting assumes that there are no explicit supersymmetric masses $\sim \int d^{2} \theta \mu^{\prime} H H^{c}$ or holomorphic Kähler potential operators $\sim \int d^{4} \theta H H^{c}$ in the hidden sector - if there are, conformal compensator effects give contributions to hiddensector parameters proportional to $m_{3 / 2}[15,16,50]$. In this case we would need $m_{3 / 2} \lesssim$ $\mathrm{GeV}$, which requires a somewhat lower cutoff, $M_{c} \lesssim 10^{14} \mathrm{GeV} .^{2}$

\subsection{Hypercharge kinetic mixing and D terms}

If there is a new $\mathrm{U}(1)_{x}$ gauge group, one can write down a renormalizable supersymmetric kinetic mixing term connecting it to hypercharge

$$
\mathcal{L} \supset \int d^{2} \theta\left(\frac{\epsilon}{2} B^{\alpha} X_{\alpha}+\frac{1}{4} B^{\alpha} B_{\alpha}+\frac{1}{4} X^{\alpha} X_{\alpha}\right)+\text { h.c. }
$$

Such a term will be generated radiatively when there are fields charged under both $\mathrm{U}(1)_{x}$ and $\mathrm{U}(1)_{Y}[52-56]$

$$
\Delta \epsilon(\mu) \simeq \frac{g_{x}(\mu) g_{Y}(\mu)}{16 \pi^{2}} \sum_{i} x_{i} Y_{i} \ln \left(\frac{\Lambda^{2}}{\mu^{2}}\right)
$$

where $x_{i}$ and $Y_{i}$ denote the charges of the $i$-th field, $\Lambda$ is the UV cutoff scale, and the log is cut off below $\mu \simeq m_{i}$, where $m_{i}$ is the mass of the $i$-th field. This leads to values of the kinetic mixing in the typical range $\epsilon \simeq 10^{-4}-10^{-2}$. Conversely, the kinetic mixing parameter $\epsilon$ can be highly suppressed or absent if there exist no such bi-fundamentals, if the underlying gauge structure consists of a simple group, or in the context of certain string-theoretic constructions [54, 55].

If a gauge kinetic mixing term is generated, it will communicate visible-sector supersymmetry breaking effects to the hidden sector. This is evident if we shift the basis of gauge fields to eliminate the gauge kinetic mixing,

$$
V_{x} \rightarrow c_{\epsilon} V_{x}, \quad V_{Y} \rightarrow V_{Y}-s_{\epsilon} V_{x},
$$

\footnotetext{
${ }^{2}$ In order to maintain a perturbative description, this may require a mild $O(10-100)$ hierarchy between the cutoff of the higher-dimensional effective field theory, $\Lambda$, and the higher-dimensional Planck scale [51].
} 
where $V_{x}$ and $V_{Y}$ are the $\mathrm{U}(1)_{x}$ and $\mathrm{U}(1)_{Y}$ gauge multiplets and

$$
s_{\epsilon} \equiv \frac{\epsilon}{\sqrt{1-\epsilon^{2}}}, \quad c_{\epsilon} \equiv \frac{1}{\sqrt{1-\epsilon^{2}}} .
$$

Doing so, one finds that the visible-sector fields acquire effective $\mathrm{U}(1)_{x}$ charges,

$$
x_{i}^{\mathrm{eff}}=-s_{\epsilon} \frac{g_{Y}}{g_{x}} Y_{i}
$$

The visible-sector fields then act as gauge messengers to the hidden $\mathrm{U}(1)_{x}$ sector [6]. We will discuss this in more detail below.

However, a usually more important effect on the $\mathrm{U}(1)_{x}$ sector comes from the hypercharge $D$ term, which induces an effective Fayet-Illiopoulos term [57] in the hidden sector $[11,12,54,58]$. A hypercharge $D$ term arises when the visible-sector Higgs fields acquire VEVs with $\tan \beta \neq 1$ induced by SUSY breaking ${ }^{3}$

$$
\xi_{Y}=-\frac{g_{Y}}{2} c_{2 \beta} v^{2}
$$

The hypercharge FI term can also receive contributions proportional to supersymmetry breaking when $\operatorname{Tr}\left(Y m^{2}\right) \neq 0 .{ }^{4}$ This vanishes in pure gauge mediation with messenger parity, but can be generated if there are violations of messenger parity [60], such as can arise if the Higgs fields couple directly to the gauge messengers [61].

Including the effect of the hypercharge FI term, the $\mathrm{U}(1)_{x} D$-term potential is given by

$$
V_{D}=\frac{g_{x}^{2}}{2} c_{\epsilon}^{2}\left(\sum_{i} x_{i}\left|\phi_{i}\right|^{2}-\frac{\epsilon}{g_{x}} \xi_{Y}\right)^{2},
$$

where $\phi_{i}$ represents a hidden-sector scalar field with $\mathrm{U}(1)_{x}$ charge $x_{i}$, while the hypercharge $D$-term potential retains its usual form. Thus, we see that the hypercharge $D$ term induces an effective $\mathrm{FI}$ term for $\mathrm{U}(1)_{x}$.

When the FI term dominates the dynamics in the hidden sector, it can induce a hidden-sector VEV

$$
\left\langle\phi_{i}\right\rangle \simeq\left(\frac{\epsilon \xi_{Y}}{g_{x} x_{i}}\right)^{1 / 2}
$$

for one or several of the scalars. This is close to the GeV scale for natural values of $\epsilon \sim 10^{-4}-10^{-3}$. Alternatively, the hypercharge FI term can be thought of as a contribution to the hidden sector scalar masses in the amount

$$
\Delta m_{\phi_{i}}^{2}=-\epsilon c_{\epsilon}^{2} g_{x} x_{i} \xi_{Y} .
$$

This contribution will be in addition to any supersymmetric or soft supersymmetrybreaking scalar masses present in the hidden sector. Let us emphasize, however, that even though the induced FI term is generated (in part) by supersymmetry breaking, and can itself trigger spontaneous supersymmetry breaking in the $\mathrm{U}(1)_{x}$ sector, it is itself a supersymmetric coupling.

\footnotetext{
${ }^{3}$ EWSB at the supersymmetric level necessarily has $\tan \beta=1$ to ensure $D$-flatness [59].

${ }^{4}$ Of course, there could be a supersymmetric contribution to $\xi_{Y}$, but this leads to the hierarchy problem of why $\xi_{Y} \ll M_{\mathrm{Pl}}$.
} 


\subsection{Little gauge mediation}

If supersymmetry breaking is communicated to the visible sector by gauge mediation involving only the MSSM gauge interactions, soft terms will also be generated for the fields in the hidden $\mathrm{U}(1)_{x}$ sector if there is gauge kinetic mixing. The typical size of such terms is less than or on the order of $m_{\mathrm{soft}}^{\text {hid }} \sim \epsilon m_{\mathrm{soft}}^{\mathrm{vis}}$, which is close to a $\mathrm{GeV}$ for $\epsilon \sim 10^{-3}$ and $m_{\mathrm{soft}}^{\mathrm{vis}} \sim \mathrm{TeV}$. Following ref. [9], we call this mechanism little gauge mediation.

The hidden-sector soft terms that arise from integrating out the gauge messengers can be computed diagrammatically or (to leading order in $F / M$ ) through the method of analytic continuation into superspace $[62,63]$. We present a derivation using the analytic continuation technique in appendix A. The leading order result for the soft scalar masses at the gauge messenger scale $M$ is

$$
m_{\phi_{i}}^{2}=\epsilon^{2} x_{i}^{2}\left(\frac{g_{x}}{g_{Y}}\right)^{2} m_{E^{c}}^{2}
$$

with all quantities evaluated at the messenger scale $M$. Let us emphasize that this result holds both for minimal and general [64] gauge mediation scenarios.

The $\mathrm{U}(1)_{x}$ gaugino soft mass is a bit more involved since the kinetic mixing in eq. (2.5) also generates an off-diagonal gaugino kinetic term. In the basis where the kinetic mixing appears explicitly and the visible-sector fields carry no $\mathrm{U}(1)_{x}$ charges, the $\left(\lambda_{Y}, \lambda_{x}\right)$ gaugino mass matrix at the messenger scale at one-loop is simply given by

$$
M_{\text {gaugino }}=\left(\begin{array}{cc}
M_{1} & 0 \\
0 & 0
\end{array}\right),
$$

where $M_{1}$ is the standard gauge-mediated contribution to the hypercharge gaugino mass. That there is no explicit $\mathrm{U}(1)_{x}$ gaugino mass generated can be understood as an example of the "gaugino screening" effect discussed in ref. [63]. In the field basis where the gaugino kinetic mixing is eliminated by the transformation of eq. (2.7), the mass matrix becomes

$$
M_{\text {gaugino }}=\left(\begin{array}{cc}
M_{1} & -s_{\epsilon} M_{1} \\
-s_{\epsilon} M_{1} & s_{\epsilon}^{2} M_{1}
\end{array}\right) \text {, }
$$

which clearly also contains a zero eigenvalue.

As in gauge mediation to the visible sector, hidden-sector $A$ - and $B$-terms are generated at the two-loop level in the presence of corresponding hidden-sector trilinear or bilinear couplings. These terms will be generated at the messenger scale with size $\sim \frac{\epsilon^{2}}{(4 \pi)^{4}} m_{\mathrm{soft}}^{\mathrm{vis}}$, and will generally be subdominant relative to renormalization group effects, which we discuss below. Note that, together with our result for the $\mathrm{U}(1)_{x}$ gaugino soft mass, we find that all $\mathrm{U}(1)_{R}$-breaking soft terms generated by little gauge mediation in the hidden sector are suppressed by a least a factor of $\epsilon$ relative to the $\mathrm{U}(1)_{R}$-preserving scalar soft masses. We will see in the following section that this feature can have important implications for the hidden-sector phenomenology. 


\subsection{Little gaugino mediation}

A GeV-scale hidden sector can also arise in a natural way if supersymmetry breaking is communicated to the visible sector by the MSSM gauginos [40, 41]. Gaugino mediation can arise in a sequestered extra-dimensional scenario where the MSSM chiral multiplets as well as the entire $\mathrm{U}(1)_{x}$ sector (gauge and chiral multiplets) are confined to a brane, with supersymmetry breaking confined to a separate brane. Sequestering can also be induced by approximately conformal strong dynamics [42], and spectra similar to that of gaugino mediation can arise from coupling gauge mediation to a conformal hidden sector [44, 45].

Allowing only the MSSM vector multiplets to propagate in the bulk between the branes, the leading-order soft terms consist of MSSM gaugino soft masses, with all other soft terms vanishing up to small corrections. The gaugino masses arise from brane-localized higher dimension operators of the form $[40,41]$

$$
\mathcal{L} \supset \int d^{2} \theta\left(\frac{M_{c}}{\Lambda}\right) \frac{1}{M_{c}} X W^{\alpha} W_{\alpha},
$$

where $\Lambda$ is the cutoff of the higher-dimensional theory and $M_{c}$ is the compactification scale. Such operators generate visible-sector gaugino masses at the compactification scale $M_{c}$ on the order of

$$
M_{a} \sim g_{a}^{2}\left(\frac{M_{c}}{\Lambda}\right) \frac{F}{M_{c}} .
$$

In this scenario, the $\mathrm{U}(1)_{x}$ gaugino will have an approximately vanishing soft mass at the scale $M_{c}$ provided it is sequestered on the MSSM brane. The leading soft terms in the fourdimensional low-energy effective theory in both the MSSM chiral and $\mathrm{U}(1)_{x}$ sectors will then arise from renormalization group running (which we describe below), and additional supergravity effects.

An additional possibility in gaugino mediation that is consistent with flavor constraints is that the MSSM Higgs chiral multiplets are allowed to propagate in the bulk $[40,41$, 65]. This can be important for the hidden sector in that it allows for the possibility of a non-vanishing hypercharge $D$ term generated by having $\left(m_{H_{u}}^{2}-m_{H_{d}}^{2}\right) \neq 0$ at the compactification scale. RG running will then provide an additional contribution to $\xi_{Y}$, beyond that induced by the Higgs VEVs.

In fact, without specifying the complete UV and GUT structure, it may be possible to simply write down the brane-localized FI-term operator

$$
\mathcal{L} \supset \int d^{2} \theta \frac{1}{\Lambda^{2}} X^{\dagger} X D_{\alpha} B^{\alpha}
$$

which is gauge invariant and yields an FI term proportional to supersymmetry breaking

$$
\xi \sim\left(\frac{F}{\Lambda}\right)^{2} \sim M_{1}^{2}
$$

which is the same order as the visible-sector hypercharge gaugino mass. While it would be interesting to further explore which UV structures may generate this operator (necessarily involving GUT-breaking effects), we postpone this to future work. 


\subsection{MSSM renormalization group effects}

In addition to the soft terms generated at the messenger threshold scale $M$ or compactification scale $M_{c}$, the visible-sector states will themselves act as messengers to the hidden sector. These effects are captured in the renormalization group (RG) equations of the hidden-sector soft parameters. They are particularly important in relation to $\mathrm{U}(1)_{R^{-}}$ breaking in the hidden sector. Indeed, a remarkable property of little gauge (and gaugino) mediation is that it shields the hidden sector from $\mathrm{U}(1)_{R^{-}}$-symmetry breaking by at least a factor of $\epsilon$. If there is no source of explicit supersymmetric $\mathrm{U}(1)_{R}$ breaking in the hidden sector, a light pseudo- $R$-axion can emerge if the $\mathrm{U}(1)_{R}$ is broken spontaneously by hidden-sector VEVs. In addition, it is even possible for hidden sectors to be approximately supersymmetric prior to gauge-symmetry breaking, in which case a pseudo-Goldstino can emerge if supersymmetry is spontaneously broken. In these situations, RG running (or possibly supergravity effects) can provide the dominant source of $\mathrm{U}(1)_{R}$ breaking, thereby setting the mass of the light state.

The leading RG effects of the visible-sector soft terms on the hidden sector come from the hypercharge gaugino mass $M_{1}$. Soft scalar masses in the hidden sector receive a contribution [66]

$$
(4 \pi)^{2} \frac{d}{d t} m_{i}^{2}=s_{\epsilon}^{2}\left(-8 x_{i}^{2} g_{x}^{2}\left|M_{1}\right|^{2}\right)+\ldots,
$$

where this is in addition to the standard contributions to the $\beta$-function from hidden-sector interactions. This term can lead to an $O(1)$ correction to the soft masses generated at the messenger scale if there is a moderate amount of running.

Perhaps more importantly, RG effects proportional to $M_{1}$ generate $R$-symmetry breaking $A$ - and $B$-terms in the presence of supersymmetric hidden-sector bilinear and trilinear interactions. These are generated as [66]

$$
\begin{aligned}
(4 \pi)^{2} \frac{d}{d t} b^{i j} & =-s_{\epsilon}^{2} M^{i j}\left[4\left(x_{i}^{2}+x_{j}^{2}\right) g_{x}^{2} M_{1}\right]+\ldots \\
(4 \pi)^{2} \frac{d}{d t} a^{i j k} & =-s_{\epsilon}^{2} y^{i j k}\left[4\left(x_{i}^{2}+x_{j}^{2}+x_{k}^{2}\right) g_{x}^{2} M_{1}\right]+\ldots,
\end{aligned}
$$

where the hidden-sector superpotential is taken to be $W_{\text {hidden }}=\frac{1}{2} M^{i j} \Phi_{i} \Phi_{j}+\frac{1}{6} y^{i j k} \Phi_{i} \Phi_{j} \Phi_{k}$ and the soft parameters defined as $V_{\text {hidden }} \supset-\left(\frac{1}{2} b^{i j} \phi_{i} \phi_{j}+\frac{1}{6} a^{i j k} \phi_{i} \phi_{j} \phi_{k}+h . c.\right)$. Note that the mass parameters generated in this way are suppressed by a factor of $\epsilon$ relative to the soft scalar masses.

\subsection{Additional mediator fields}

Finally, there may exist additional fields in the low-energy spectrum which mediate between the visible and hidden sectors. Among the many possibilities, we will focus on two cases: bi-fundamentals charged under both the visible and hidden gauge groups, and singlets that couple directly to fields in both sectors. In the sections that follow, we will construct explicit examples that realize both cases.

Light bi-fundamentals in the spectrum, charged under both the visible-sector gauge group and $\mathrm{U}(1)_{x}$, act as gauge messengers to the hidden sector. Such bi-fundamentals 
develop soft masses from gauge or gaugino mediation, and then pass on this supersymmetry breaking through gauge loops connecting them to the rest of the $\mathrm{U}(1)_{x}$ sector. The relevant diagrams are analogous to those arising in standard gauge-mediated models (with the bi-fundamentals as messengers), with the important difference that the supertrace of the bi-fundamental multiplets may be non-vanishing. The corresponding generalization of minimal gauge mediation is worked out in ref. [67]. Hidden-sector scalar soft masses are generated on the order of

$$
\Delta m_{i}^{2} \sim-\frac{2 g_{x}^{4} x_{i}^{2}}{(4 \pi)^{4}} \operatorname{Str}\left(x_{b f}^{2} M_{b f}^{2}\right) \ln \left(\frac{\Lambda^{2}}{M_{b f}^{2}}\right),
$$

where $x_{i}$ is the $\mathrm{U}(1)_{x}$ charge, $M_{b f}$ denotes the bi-fundamental mass matrix, and $\Lambda$ is approximately the scale at which a vanishing supertrace is restored. Up to the logarithmic (running) enhancement, this leads to soft masses for the pure $\mathrm{U}(1)_{x}$ states suppressed by a loop factor relative to the visible sector.

Bi-fundamentals also can contribute to $A$ - and $B$-terms as well as the $\mathrm{U}(1)_{x}$ gaugino mass, with a necessary condition being that their soft parameters contain $\mathrm{U}(1)_{R}$ breaking. For bi-fundamental couplings of the form

$$
\begin{aligned}
W & \supset \mu_{F} F F^{c} \\
V_{\text {soft }} & \supset m_{F}^{2}|F|^{2}+m_{F^{c}}^{2}\left|F^{c}\right|^{2}-\left[(B \mu)_{F} F F^{c}+h . c .\right],
\end{aligned}
$$

the contribution to the $\mathrm{U}(1)_{x}$ gaugino mass at the scale $\mu_{F}<M_{\text {mess }}$ in the limit of $\mu_{F}^{2} \gg$ $m_{F}^{2}=m_{F^{c}}^{2} \gtrsim(B \mu)_{F}$ is typically on the order of

$$
M_{x} \simeq \frac{g_{x}^{2} x_{F}^{2}}{8 \pi^{2}} \frac{(B \mu)_{F}}{\mu_{F}}
$$

with the general expression for the gaugino mass given in ref. [67]. An important special case occurs when $\mu_{F}$ is a genuinely supersymmetric threshold (i.e., the $(B \mu)_{F}$ term arises entirely from supersymmetry breaking contained in the wavefunction renormalization of $F$ and $F^{c}$ ). With this provision, the gaugino screening theorem of ref. [63] implies that the contributions to $M_{x}$ cancel at this loop order, leading to a highly suppressed gaugino mass.

Bi-fundamental fields in the spectrum will also induce (or add to) kinetic mixing between $\mathrm{U}(1)_{x}$ and hypercharge, which in turn will further mediate supersymmetry breaking to the hidden sector. In fact, this scenario is continuously connected to the situation considered previously, where all bi-fundamentals were assumed to have been integrated out above the scale of the gauge messengers or the compactification scale. The only difference is that we have now lowered the bi-fundamental mass scale below the messenger scale. Even so, let us also mention that the additional logarithmic enhancement in $\epsilon$ due to lighter bi-fundamentals typically requires relatively small values of the $\mathrm{U}(1)_{x}$ gauge coupling $g_{x} \lesssim 0.1$ (assuming order unity charges) to avoid generating an unacceptably large low-scale value of $\epsilon$.

Supersymmetry breaking can also be mediated to the hidden sector by gauge-singlet chiral superfields. As an example, consider the gauge singlet $S$ with superpotential cou- 
plings

$$
W \supset \zeta S H_{u} H_{d}+\lambda S H H^{c}
$$

Here, $H_{u}$ and $H_{d}$ are the MSSM Higgs multiplets and $H$ and $H^{c}$ are a vector pair of states charged under $\mathrm{U}(1)_{x}$. The singlet $S$ may or may not condense to generate the $\mu$ term. The interactions of eq. (2.27) will then generate a running contribution to the soft scalar masses of $H$ and $H^{c}$,

$$
\begin{gathered}
(4 \pi)^{2} \frac{d}{d t} m_{S}^{2}=4|\zeta|^{2}\left(m_{S}^{2}+m_{H_{u}}^{2}+m_{H_{d}}^{2}+\left|A_{\zeta}\right|^{2}\right) \\
+2|\lambda|^{2}\left(m_{S}^{2}+m_{H}^{2}+m_{H^{c}}^{2}+\left|A_{\lambda}\right|^{2}\right)+\ldots \\
(4 \pi)^{2} \frac{d}{d t} m_{H^{(c)}}^{2}=2|\lambda|^{2}\left(m_{S}^{2}+m_{H}^{2}+m_{H^{c}}^{2}+\left|A_{\lambda}\right|^{2}\right)+\ldots
\end{gathered}
$$

At the messenger or compactification scale, the Higgs soft masses are typically positive. The singlet soft mass, which vanishes at the input scale in minimal scenarios, is then driven negative. This in turn drives the soft masses for $H$ and $H^{c}$ positive. As long as $\lambda$ or $\zeta$ are somewhat small, this can lead to $\mathrm{GeV}$-scale contributions to hidden-sector parameters.

Alternatively, $S$ can couple directly to the supersymmetry-breaking sector and pick up a large soft mass that then drives the $H$ and $H^{c}$ masses negative. This can in turn cause the hidden-sector gauge group to break. An additional possibility is that the $H$ and $H^{c}$ fields receive weak-scale masses and supersymmetry-breaking parameters through larger couplings and possible (auxiliary) VEVs of $H_{u}, H_{d}$, and $S$. In this case, they play a similar role as the bi-fundamentals considered above, mediating supersymmetry breaking to the rest of hidden sector.

\section{Models}

We present here a handful of simple models for the hidden $\mathrm{U}(1)_{x}$ sector that illustrate the supersymmetry-breaking mechanisms discussed in section 2 . In many cases, the models we consider run into problems with constraints from nucleosynthesis or generate dark matter in excess of the observed density. As such, our main goal will be to construct simple viable hidden sectors that avoid these difficulties. We will see that, because of these constraints, it is generally favorable for the gravitino mass $m_{3 / 2}$ to be heavier than the lightest hiddensector particle (LHP). We begin with a simple little-gauge-mediated model whose matter content is a pair of hidden Higgs fields with a bare $\mu^{\prime}$ term. We will see that this model generically contains a light fermion with an overly large relic density unless supergravity effects push its mass above that of the $\mathrm{U}(1)_{x}$ gauge boson. This regime also gives a natural explanation of the origin of the $\mu^{\prime}$ term, but has the disadvantage that the spectrum depends on unknown UV physics.

We then turn to a hidden NMSSM model which promotes the $\mu^{\prime}$ term to a hidden singlet. When the gravitino is lighter than the LHP, the scenario is only viable for a very low supersymmetry-breaking scale, or if additional operators allow the LHP to decay. We will see that making the gravitino heavier allows for additional viable scenarios as long 
as the LHP retains a large enough annihilation channel. An interesting possibility for doing this is to take $m_{3 / 2} \sim m_{\text {hid }}$ while sequestering supergravity effects. In this case the LHP still has phase space to annihilate to nearly degenerate gauge bosons, with the mass splitting set by anomaly mediation effects. The LHP is then a viable candidate to be a component of the dark matter.

Lastly, we discuss models where the mediation occurs via dynamical matter fields, and construct an explicit model for singlet mediation and mediation by bi-fundamentals. The singlet scenario often contains a light pseudo-axion, but can be viable provided the axion is able to decay through small couplings to the visible sector. With bi-fundamentals, the low-energy spectrum and phenomenology is frequently very similar to the case of little gauge mediation.

\subsection{Minimal $\mu^{\prime}$ model}

The minimal viable $\mathrm{U}(1)_{x}$ dark hidden sector consists of a vector-like pair of chiral multiplets $H$ and $H^{c}$ with the superpotential

$$
W \supset \mu^{\prime} H H^{c}
$$

The most natural values of $\mu^{\prime}$ are either $m_{3 / 2}$ or 0 (or very large!), and we include in this class of models the limit $\mu^{\prime} \rightarrow 0$. To permit symmetry breaking, $\mu^{\prime}$ must not be much larger than other contributions to hidden-sector parameters. For the time being we leave the origin of $\mu^{\prime}$ unspecified, although we will comment on how values of $\mu^{\prime} \lesssim \mathrm{GeV}$ can arise naturally from supergravity effects when $m_{3 / 2} \sim \mathrm{GeV}$. In analyzing this model, we will assume that $\mathrm{U}(1)_{x}$ has kinetic mixing with hypercharge as discussed above.

In the absence of supersymmetry breaking, the tree-level low-energy scalar potential of the theory is given by

$$
\begin{aligned}
V= & \left|\mu^{\prime}\right|^{2}\left(|H|^{2}+\left|H^{c}\right|^{2}\right) \\
& +\frac{g_{x}^{2}}{2}\left(x_{H}|H|^{2}-x_{H}\left|H^{c}\right|^{2}-\xi\right)^{2} .
\end{aligned}
$$

The parameter $\xi$ appearing in the $D$-term part of the potential arises from the hypercharge FI term set by the VEV of the MSSM Higgs fields, and is given by

$$
\xi \equiv \frac{\epsilon}{g_{x}} \xi_{Y}=-\frac{\epsilon}{g_{x}} \frac{g_{Y}}{2} c_{2 \beta} v^{2} .
$$

This term will receive additional contributions if there is a non-vanishing FI term for either hypercharge or $\mathrm{U}(1)_{x}$, or if $\operatorname{Tr}\left(Y m^{2}\right) \neq 0$.

Without loss of generality, we assume $x_{H}>0$ and $\epsilon>0$ so that $\xi$ is positive and the potential gives $H$ a negative mass-squared contribution. With these sign conventions, the minimum of the potential lies at

$$
\langle H\rangle \equiv \eta=\sqrt{\xi / x_{H}-\left|\mu^{\prime}\right|^{2} /\left(g_{x} x_{H}\right)^{2}}, \quad\left\langle H^{c}\right\rangle=0 .
$$

Thus, even in the absence of soft supersymmetry-breaking terms in the hidden sector, the gauge symmetry (as well as supersymmetry) is spontaneously broken. 
The corresponding spectrum of bosonic states then consists of a massive gauge boson and a physical Higgs boson $h$ derived from $H$, both with mass

$$
m_{h}^{2}=m_{Z_{x}}^{2}=2 g_{x}^{2} x_{H}^{2} \eta^{2} .
$$

as well as a complex scalar derived from $H^{c}$ with mass $2\left|\mu^{\prime}\right|^{2}$. For $g_{x} x_{H}=0.1, \epsilon=10^{-3}$, and $\xi$ generated only by the MSSM Higgs VEVs, we find $m_{Z_{x}} \lesssim 1 \mathrm{GeV}$. As $\mu^{\prime} \rightarrow 0$, the potential acquires a flat direction at tree-level corresponding to this state becoming massless and supersymmetry in the hidden sector being restored.

Among the fermionic states, two are comprised of a Dirac mixture of the Higgsinos and the $\mathrm{U}(1)_{x}$ gaugino with mass

$$
M_{2,3}^{f}=\sqrt{\left|\mu^{\prime}\right|^{2}+m_{Z_{x}}^{2}}
$$

The third fermionic state is a massless Weyl fermion. It is the Goldstino corresponding to the spontaneous breaking of supersymmetry in this sector.

The spectrum will be deformed by the inclusion of soft supersymmetry-breaking operators. The precise effect of the soft terms depends on their origin, whether from gauge or gaugino mediation or due to residual supergravity effects. In general, however, the phenomenology of the hidden sector can be classified according to the scale of the hiddensector masses relative to the gravitino mass $m_{3 / 2}$. Thus, we consider the distinct cases $m_{3 / 2} \ll m_{\text {hid }}$ and $m_{3 / 2} \gtrsim m_{\text {hid }}$.

\subsection{1 $\mathrm{m}_{3 / 2} \ll \mathrm{m}_{\text {hid }}$}

Such a hierarchy can arise with a messenger or compactification scale well below $10^{14} \mathrm{GeV}$ in gauge or gaugino mediation. Little gauge or gaugino mediation effects then provide the dominant contributions to the soft terms, which are parametrically smaller by a power of $\sqrt{\epsilon}$ compared to the symmetry breaking induced by $\xi$. We can thus treat their effects as small perturbations on the supersymmetric spectrum described above.

The tree-level scalar potential becomes

$$
\begin{aligned}
V= & \left(m_{H}^{2}+\left|\mu^{\prime}\right|^{2}\right)|H|^{2}+\left(m_{H^{c}}^{2}+\left|\mu^{\prime}\right|^{2}\right)\left|H^{c}\right|^{2} \\
& -\left(B \mu^{\prime} H H^{c}+\text { h.c. }\right) \\
& +\frac{g_{x}^{2}}{2}\left(x_{H}|H|^{2}-x_{H}\left|H^{c}\right|^{2}-\xi\right)^{2} .
\end{aligned}
$$

For the time being, we will assume that the $\mu^{\prime}$ term is genuinely supersymmetric in origin and neglect supergravity effects. The $B \mu^{\prime}$ term is then generated within little gauge or gaugino mediation primarily through RG running as in eq. (2.22), on the order of $B \mu^{\prime} \lesssim$ $g_{x}^{2} \epsilon^{2} \mu^{\prime} M_{1}$, and is parametrically smaller than the scalar soft masses by a factor of $\epsilon$, which in turn are smaller than the symmetry breaking induced by the FI term by a factor of $\sqrt{\epsilon}$. This has important implications for the spectrum and phenomenology of the model.

With the inclusion of soft terms, the VEV of $H$ is shifted to

$$
\langle H\rangle \equiv \eta=\sqrt{\xi / x_{H}-\left(m_{H}^{2}+\left|\mu^{\prime}\right|^{2}\right) /\left(x_{H} g_{x}\right)^{2}} .
$$


The $B \mu^{\prime}$ term forces the $H^{c}$ field to develop a VEV as well. Since this term is subleading, the corresponding VEV of $H^{c}$ is much smaller than that of $H$. Denoting the ratio of VEVs by $\tan \alpha=\left\langle H^{c}\right\rangle /\langle H\rangle$, we find

$$
\tan \alpha \simeq \frac{B \mu^{\prime}}{m_{H}^{2}+m_{H^{c}}^{2}+2\left|\mu^{\prime}\right|^{2}}
$$

With our assumption of a supersymmetric origin of the $\mu^{\prime}$ term, this ratio is on the order of $\tan \alpha \sim \epsilon^{2} M_{1} /\left|\mu^{\prime}\right|$.

The bosonic spectrum still contains a single real Higgs scalar that is degenerate (at tree-level) with the gauge boson with mass given again by eq. (3.5), as well as CP-even and CP-odd scalars derived primarily from $H^{c}$ with masses

$$
m_{h^{c}}^{2} \simeq m_{a^{c}}^{2} \simeq m_{H}^{2}+m_{H^{c}}^{2}+2\left|\mu^{\prime}\right|^{2} .
$$

These states are split slightly by the $B \mu^{\prime}$ term.

The fermion mass matrix becomes

$$
\mathcal{M}^{f} \simeq\left(\begin{array}{ccc}
0 & -\sqrt{2} x_{H} g_{x} \eta \tan \alpha \sqrt{2} x_{H} g_{x} \eta \\
-\sqrt{2} x_{H} g_{x} \eta \tan \alpha & 0 & \mu^{\prime} \\
\sqrt{2} x_{H} g_{x} \eta & \mu^{\prime} & 0
\end{array}\right) .
$$

Two of the mass eigenvalues are relatively heavy, and correspond to a nearly Dirac state with mass given by eq. (3.6). The third fermion state is much lighter, and coincides with the massless Goldstino fermion found above. Explicit supersymmetry and $R$-symmetry breaking in the form of the subleading $B \mu^{\prime}$ term (or gaugino mass $M_{x}$ ) is required to lift this state. Including the effect of $B \mu^{\prime}$, the light mass is

$$
M_{1}^{f} \simeq\left(\frac{2 m_{Z_{x}}^{2}}{m_{Z_{x}}^{2}+\left|\mu^{\prime}\right|^{2}}\right) \mu^{\prime} \tan \alpha \sim \epsilon^{2} g_{x}^{2} M_{1}
$$

For $M_{1} \sim 100 \mathrm{GeV}, \mu^{\prime} \sim \mathrm{GeV}, \epsilon \sim 10^{-3}$, this is on the order of $0.1 \mathrm{MeV}$.

The very light pseudo-Goldstino fermion state is evidently the lightest hidden particle (LHP) in this sector. It is metastable due to $R$-parity (whose conservation requires $H$ and $H^{c}$ to be even), and is only able to decay or annihilate into visible sector states through kinetic mixing. We estimate the corresponding annihilation cross-section through $s$-channel gauge boson exchange into $e^{+} e^{-}\left(M_{1}^{f}>m_{e}\right)$ or $\nu \bar{\nu}\left(M_{1}^{f}<m_{e}\right)$ to be [6]

$$
\langle\sigma v\rangle \simeq\left\{\begin{array}{l}
\frac{g_{x}^{2} x_{H}^{2} e^{2} c_{W}^{2}}{3 \pi}\left|U_{f x}\right|^{4} \epsilon^{2} \frac{\left(M_{1}^{f}\right)^{2}}{m_{Z x}^{4}} v_{f . o .}^{2} \sim \frac{g_{x}^{2} x_{H}^{2} e^{2} c_{W}^{2}}{12 \pi}\left|U_{f x}\right|^{4} \epsilon^{6} \frac{M_{1}^{2}}{\eta^{4}} v_{f . o .}^{2}, M_{1}^{f}>m_{e}, \\
\frac{g_{x}^{2} x_{H}^{2} g^{\prime 2}}{4 \pi}\left|U_{f x}\right|^{4} \epsilon^{2} \frac{\left(M_{1}^{f}\right)^{6}}{m_{Z_{x}}^{4} m_{Z}^{4}} v_{f . o .}^{2} \sim \frac{g_{x}^{10} x_{H}^{2} g^{\prime 2}}{16 \pi}\left|U_{f x}\right|^{4} \epsilon^{14} \frac{M_{1}^{6}}{\eta^{4} m_{Z}^{4}} v_{f . o .}^{2}, M_{1}^{f}<m_{e},
\end{array}\right.
$$

where $v_{f . o .}$ is the typical velocity during freeze-out and $U_{f x}$ is the gaugino fraction of the state. The additional suppression for $M_{1}^{f}<m_{e}$ comes from the fact that the annihilation final state consists of neutrinos. For $m_{Z_{x}}^{2} \ll m_{Z}^{2}$, the $\mathrm{U}(1)_{x}$ mixes primarily with electromagnetism and couplings to neutrinos (due to a residual mixing with the $Z^{0}$ ) are 
suppressed [11]. This cross-section is much too small to yield an acceptable relic density, even with $M_{1}^{f}>m_{e}$. Indeed, for $M_{1}^{f}<m_{e}$, the light fermion state decouples while it is still relativistic.

Under our assumption of a very light gravitino, the light fermion is unstable to decay into a photon and a gravitino via its $\epsilon$-mediated mixing with the photino. The corresponding lifetime is

$$
\begin{aligned}
\tau & =\frac{16 \pi\langle F\rangle^{2}}{m_{\chi}^{5}\left|P_{\gamma}\right|^{2}} \\
& \simeq\left(3 \times 10^{23} s\right)\left(\frac{\sqrt{\langle F\rangle}}{100 \mathrm{TeV}}\right)^{4}\left(\frac{0.1 \mathrm{MeV}}{m_{\chi}}\right)^{5}\left(\frac{\epsilon}{\left|P_{\gamma}\right|}\right)^{2}\left(\frac{10^{-3}}{\epsilon}\right)^{2},
\end{aligned}
$$

where $F$ is the auxiliary VEV parametrizing the underlying source of supersymmetry breaking in all sectors, and $P_{\gamma} \sim \epsilon$ is the projection of the light $\mathrm{U}(1)_{x}$-sector fermion onto the photino. This lifetime is on the order of the age of the universe. Since the relic density of the light state is too large, this scenario is ruled out as it stands.

So far, we have completely neglected supergravity contributions to the hidden-sector soft parameters. However, even though we have assumed $m_{3 / 2} \ll m_{\text {hid }}$, we could still have $m_{3 / 2}^{2} \gtrsim g_{x}^{2} \epsilon^{2}\left|\mu^{\prime}\right| M_{1}$, in which case residual supergravity effects would provide the dominant contribution to $B \mu^{\prime}$ (and $M_{x}$ ). The primary effect would be to push up the mass of the light fermion, which would weigh nearly the same as the gravitino. If the light fermion is lighter than the gravitino it is then stable, and will still generically have too large of a relic density. In either case, whether the gravitino is lighter than the lightest fermion in the hidden sector (in which case the fermion decays to the gravitino after BBN) or if the gravitino is heavier than the fermion (in which case the fermion has too high a relic density), this scenario is not viable.

\subsection{2 $\mathrm{m}_{3 / 2} \gtrsim \mathrm{m}_{\text {hid }}$}

Our results lead us to consider larger values of $m_{3 / 2}$, such as might emerge with a messenger or compactification scale on the order of or greater than $M \sim 10^{14} \mathrm{GeV}$ in gauge or gaugino mediation. Indeed, the case of gauge mediation with $m_{3 / 2} \sim 1 \mathrm{GeV}$ arises in sweet-spot models of supersymmetry breaking [68], as well as in certain GUT constructions based on $F$-theory [69-72]. Residual supergravity-induced flavor-mixing effects in this case lie on the border of what is consistent with current constraints [49]. The precise effect on the hidden sector spectrum for a given value of $m_{3 / 2}$ depends on whether or not supergravity-mediated effects are sequestered. We consider both possibilities here.

In the case of gauge mediation without sequestering, residual supergravity-mediated effects will generate contributions to all soft masses on the order of $m_{3 / 2}$. Thus, if the hidden sector is to be light (and to avoid flavor constraints) we should have $m_{3 / 2} \lesssim \mathrm{GeV}$. For $m_{3 / 2} \sim \mathrm{GeV}$ supergravity contributions to the hidden-sector soft terms will be of the same order as those due to kinetic mixing. A value of $\mu^{\prime} \sim \sqrt{\left|B \mu^{\prime}\right|} \sim m_{3 / 2}$ can also arise through the standard Giudice-Masiero mechanism [50]. The precise particle spectrum will therefore depend on unknown UV physics. 
When the lightest $R$-parity odd state in the spectrum is lighter than the gravitino, it will be a stable dark matter candidate. If the gauge coupling $g_{x}$ is somewhat small, supergravity contributions can push all the gaugino and Higgsino states that could make up the stable state to be heavier than the gauge boson and the scalar Higgs $h$. This allows the lightest $R$-odd fermion to annihilate very efficiently to gauge bosons, with rate [73]

$$
\begin{aligned}
\langle\sigma v\rangle & \simeq \frac{g_{x}^{4} x_{h}^{4}}{64 \pi} \frac{\left|U_{f h}\right|^{4}}{M_{1}^{f^{2}}} \\
& \simeq\left(6 \times 10^{-24} \mathrm{~cm}^{3} / \mathrm{s}\right)\left(\frac{1 \mathrm{GeV}}{M_{1}^{f}}\right)^{2}\left(\frac{g_{x} x_{H}}{0.1}\right)^{4}\left|U_{f h}\right|^{4},
\end{aligned}
$$

where $U_{f h}$ is an order unity mixing factor into Higgsinos. One can thus easily avoid too large a relic density for the stable $R$-odd fermion.

The gauge boson and the hidden Higgs $h$ will themselves decay rapidly to the visible sector. Of these two states, the hidden Higgs $h$ will be longer-lived, with decays to photon pairs by mixing with the visible SM-like Higgs boson $h^{0}$, as well as decays to electrons through kinetic mixing. The lifetime for di-photon decays through Higgs mixing is on the order of

$$
\begin{aligned}
\tau_{h \rightarrow \gamma \gamma} & \simeq \frac{256 \pi^{3}}{\alpha^{2}} \frac{1}{\epsilon^{2}} \frac{m_{h_{0}}^{4}}{m_{h}^{5}} \\
& \simeq\left(2 \times 10^{-2} s\right)\left(\frac{10^{-3}}{\epsilon}\right)^{2}\left(\frac{m_{h^{0}}}{115 \mathrm{GeV}}\right)^{4}\left(\frac{1 \mathrm{GeV}}{m_{h}}\right)^{5} .
\end{aligned}
$$

This is typically longer than the lifetime for hidden Higgs decays to four electrons via kinetic mixing, though it goes through a higher power of $\epsilon$ :

$$
\begin{aligned}
\tau_{h \rightarrow 4 e} & \simeq \frac{256 \pi^{3}}{g_{x}^{2} x_{H}^{2} e^{4} c_{W}^{4}} \frac{1}{\epsilon^{4}} \frac{1}{m_{h}} \\
& \simeq\left(1 \times 10^{-4} s\right)\left(\frac{0.1}{g_{x} x_{H}}\right)^{2}\left(\frac{10^{-3}}{\epsilon}\right)^{4}\left(\frac{1 \mathrm{GeV}}{m_{h}}\right) .
\end{aligned}
$$

There is also a related loop-mediated decay to two electrons that we estimate to be of similar size. Thus, this scenario leads to a viable, but UV-dependent, phenomenology.

When supergravity effects are sequestered, such as is required for (high-scale) gaugino mediation to be the dominant source of the MSSM soft masses, it is possible for anomalymediated contributions to set the scale of the hidden sector, with $m_{\text {hid }} \sim m_{3 / 2} /(4 \pi)^{2}[14]$. However, in order to avoid generating too large of a hidden-sector $B \mu^{\prime}$ term, we must have $\mu^{\prime}=0$ in the superpotential and forbid the operator $H H^{c}$ in the Kähler potential. In this case, the scenario with vanishing superpotential is highly predictive assuming there are no additional non-decoupling effects. However, it has the problem that the hidden-sector potential is unstable. This is because the soft masses induced by anomaly mediation are given by

$$
m_{H}^{2}=m_{H^{c}}^{2}=-\frac{4 g_{x}^{4} x_{H}^{4}}{(4 \pi)^{4}} m_{3 / 2}^{2}<0
$$


and so the D-flat direction $\langle H\rangle=\left\langle H^{c}\right\rangle$ is not stabilized. Including an FI term in the potential does not help stabilize this direction because the sum of the squared soft masses does not change (and is still negative). Thus, this scenario requires additional contributions to the potential in order to be viable.

\subsection{Hidden sector NMSSM}

The minimal model described above contains a dimensionful coupling $\mu^{\prime}$ whose origin can be problematic (unless $m_{3 / 2} \sim m_{\text {hid }}$ ), and often gives rise to a light fermion state or an unstable potential. Both of these possible difficulties can be overcome by adding a singlet $S$ to the theory and taking the superpotential to be

$$
W \supset \lambda S H H^{c} \text {. }
$$

We can enforce this form by imposing a discrete or continuous global symmetry in addition to the gauged $\mathrm{U}(1)_{x}$. Such a symmetry can also prevent a direct coupling of the singlet $S$ to the visible-sector Higgs fields, the gauge messengers, or the supersymmetry-breaking sector.

Neglecting soft supersymmetry-breaking operators, the tree-level scalar potential in this theory is

$$
\begin{aligned}
V= & |\lambda|^{2}|H|^{2}\left(\left|H^{c}\right|^{2}+|S|^{2}\right)+|\lambda|^{2}\left|H^{c}\right|^{2}|S|^{2} \\
& +\frac{g_{x}^{2}}{2}\left(x_{H}|H|^{2}-x_{H}\left|H^{c}\right|^{2}-\xi\right)^{2},
\end{aligned}
$$

where again we take $\xi>0$ and $x_{H}>0$. The supersymmetric global minimum of this potential has $\langle H\rangle \equiv \eta \simeq \sqrt{\xi / x_{H}}$, along with $\langle S\rangle=0=\left\langle H^{c}\right\rangle$. At this minimum, the theory has an exact global U(1) symmetry under which $S$ and $H^{c}$ have opposite charges. As a result, the theory breaks into two sectors, with the lightest state in the sector derived from $S$ and $H^{c}$ absolutely stable up to explicit breaking by higher-dimensional operators. ${ }^{5}$

The spectrum of the theory within this supersymmetric minimum consists of a massive vector multiplet and a pair of chiral multiplets. This can be seen from the fermion mass matrix, which in the basis $\left(\tilde{\lambda}, \tilde{H}, \tilde{H}^{c}, \tilde{S}\right)$ is given by

$$
\mathcal{M}^{f}=\left(\begin{array}{cccc}
0 & \sqrt{2} x_{H} g_{x} \eta & 0 & 0 \\
\sqrt{2} x_{H} g_{x} \eta & 0 & 0 & 0 \\
0 & 0 & 0 & \lambda \eta \\
0 & 0 & \lambda \eta & 0
\end{array}\right)
$$

The condensing $H$ chiral multiplet gets eaten by the gauge multiplet yielding a massive vector multiplet with mass $m_{x}=\sqrt{2} g_{x} x_{H} \eta$, while the VEV of $H$ generates a joint supersymmetric mass for $H^{c}$ and $S$ producing two chiral multiplets of mass $m_{\lambda}=\lambda \eta$. The fermion states of these multiplets mix to form a single Dirac fermion (necessary on account of the accidental $\mathrm{U}(1)$ ), while the scalar states do not mix at all at tree-level.

\footnotetext{
${ }^{5}$ Including an $S^{3}$ term in the superpotential breaks the global U(1) down to a $\mathbb{Z}_{3}$ subgroup, but still gives rise to a stable state in this sector.
} 
This simple picture is deformed by adding supersymmetry-breaking soft terms to the potential. The precise effect of the soft terms depends on their origin, whether from gauge or gaugino mediation or due to residual supergravity effects. In general, however, the phenomenology of the hidden sector can be classified according to the scale of the hidden masses relative to the gravitino mass $m_{3 / 2}$. We again consider the cases $m_{3 / 2} \ll m_{\text {hid }}$ and $m_{3 / 2} \gtrsim m_{\text {hid }}$

\subsection{1 $\mathrm{m}_{3 / 2} \ll \mathrm{m}_{\text {hid }}$}

When $m_{3 / 2} \ll m_{\text {hid }}$, little gauge or gaugino mediation then provides the largest contributions to the $\mathrm{U}(1)_{x}$-sector soft terms, which are parametrically smaller than the VEV induced by $\xi$, allowing us to again treat them as small perturbations on the supersymmetric spectrum. The tree-level scalar potential then becomes

$$
\begin{aligned}
V= & \left(m_{H}^{2}-x_{H} g_{x}^{2} \xi\right)|H|^{2}+\frac{x_{H}^{2} g_{x}^{2}}{2}|H|^{4} \\
& +\left(m_{H^{c}}^{2}+x_{H} g_{x}^{2} \xi-g_{x}^{2} x_{H}^{2}|H|^{2}+|\lambda|^{2}|H|^{2}\right)\left|H^{c}\right|^{2}+\frac{x_{H}^{2} g_{x}^{2}}{2}\left|H^{c}\right|^{4} \\
& +\left(m_{S}^{2}+|\lambda|^{2}|H|^{2}\right)|S|^{2}+|\lambda|^{2}|S|^{2}\left|H^{c}\right|^{2}-\left(\lambda A_{\lambda} S H^{c} H+\text { h.c. }\right) .
\end{aligned}
$$

With $x_{H} g_{x}^{2} \xi \gg m_{H^{(c)}}^{2}$, the VEV is now shifted to

$$
\langle H\rangle \equiv \eta=\sqrt{\xi / x_{H}-m_{H}^{2} /\left(x_{H} g_{x}\right)^{2}}
$$

while $\left\langle H^{c}\right\rangle=0=\langle S\rangle$ as before. As a result, the potential maintains an exact $\mathrm{U}(1)$ global symmetry under which $H^{c}$ and $S$ are oppositely charged. The spectrum of states still consists of a massive vector multiplet and a "Dirac" pair of chiral multiplets, although the components within these multiplets will now be split in their masses.

Within the $\lambda$ sector of the theory derived from $H^{c}$ and $S$, the bosonic states consist of a complex $h^{c}$ scalar derived from $H^{c}$ with mass

$$
m_{h^{c}}^{2}=m_{H^{c}}^{2}+m_{H}^{2}+|\lambda|^{2} \eta^{2}
$$

as well as a complex $h^{s}$ scalar derived from $S$ with mass

$$
m_{h^{s}}^{2}=m_{s}^{2}+|\lambda|^{2} \eta^{2}
$$

There will be a very small additional mixing between $h^{c}$ and $h^{s}$ on the order of $A_{\lambda} \eta / m_{H}^{2} \sim$ $\sqrt{\epsilon}$ induced by the subleading $A_{\lambda}$ term. The unbroken accidental global U(1) in this sector ensures that the mixed mass eigenstates are complex scalars with degenerate real and imaginary components. This same $\mathrm{U}(1)$ also ensures that the fermion state derived from $S$ and $H^{c}$ is pure Dirac, and its tree level mass is still $m_{\lambda}=\lambda \eta$.

Among these states, the $h^{s}$ scalar will be the lightest on account of the soft mass $m_{s}^{2}$. This mass runs negative in the IR due to the effect of the $\lambda$ coupling on the RG evolution. In fact, neglecting subleading contributions to the running from the $A$-terms, it is not hard to show that (to one-loop order) we have $\left(m_{H}^{2}+m_{H^{c}}^{2}+m_{s}^{2}\right) \geq 0, m_{s}^{2} \leq 0$, and 
$m_{H}^{2}=m_{H^{c}}^{2} \geq 0$ throughout the RG flow to the IR, given little gauge or gaugino mediation boundary conditions at the messenger or compactification scale. The $h^{s}$ scalar is stable to the extent that the accidental global U(1) is not accidentally broken.

All states in the gauge sector have equal masses up to small corrections. The fermionic states retain a Dirac mass of $m_{x}=\sqrt{2} x_{H} g_{x} \eta$ but acquire a tiny $\epsilon$-suppressed Majorana splitting from the subleading gaugino soft mass. Radiative effects also split these states apart from the gauge boson by an amount on the order of $\lambda^{2} m_{s}^{2} / 16 \pi^{2}$. For example, the tree-level mass of the physical real scalar $h$ is given by

$$
m_{h}^{2}=2 x_{H}^{2} g_{x}^{2} \eta^{2}
$$

as for the fermions and the gauge boson, but is lifted by radiative corrections. The dominant effect is to raise the effective quartic coupling $Q_{\text {eff }}$ analogously to the Higgs quartic in the MSSM. This produces the shift

$$
Q_{\mathrm{eff}}=2 x_{H}^{2} g_{x}^{2} \rightarrow 2 x_{H}^{2} g_{x}^{2}+\frac{|\lambda|^{4}}{8 \pi^{2}} \ln \left(\frac{m_{h^{c}}^{2} m_{h^{s}}^{2}}{|\lambda|^{4} \eta^{4}}\right) .
$$

The logarithm is non-negative within little gauge or gaugino mediation on account of the soft masses appearing in the masses of $m_{h^{c}}^{2}$ and $m_{h^{s}}^{2}$ along with the properties of their RG flow discussed above. This pushes up the real scalar mass $m_{h}^{2}=Q_{\text {eff }} \eta^{2}$ relative to the treelevel value. The other states receive a radiative shifts in their masses of the same order. These splittings are all very small relative to the tree-level masses due to loop suppression as well as the hierarchy $m_{\text {soft }} \sim \sqrt{\epsilon} \eta$.

The phenomenology of this scenario depends primarily on which of the two sectors is lighter. Whether or not the $\lambda$ sector is lighter, the unbroken global $\mathrm{U}(1)$ ensures that the lightest $h^{s}$ scalar is stable, while the slightly heavier Dirac fermion state is metastable on account of $R$-parity (assuming all fields are $R$-even) and annihilates efficiently into the lighter scalar. When this chiral sector is lighter than the massive gauge sector, the lightest $h^{s}$ scalar can only annihilate into lighter visible sector particles through the $s$-channel exchange of a $\mathrm{U}(1)_{x}$ gauge boson. We estimate the cross-section for this process in the early universe to be [39]

$$
\begin{aligned}
\langle\sigma v\rangle \simeq & \frac{2 g_{x}^{2} x_{H}^{2} e^{2} c_{W}^{2}}{3 \pi} \frac{m_{h^{s}}^{2}}{\left(4 m_{h_{s}}^{2}-m_{Z_{x}}^{2}\right)^{2}} \epsilon^{2}\left|U_{c s}\right|^{4} v_{f . o .}^{2} . \\
\simeq\left(1 \times 10^{-36} \mathrm{~cm}^{3} / s\right)\left(\frac{g_{x} x_{H}}{0.1}\right)^{2}\left(\frac{m_{h_{s}}}{0.1 \mathrm{GeV}}\right)^{2}\left(\frac{1 \mathrm{GeV}}{m_{Z_{x}}}\right)^{4} & \quad\left(\frac{\left|U_{c s}\right|}{\sqrt{\epsilon}}\right)^{4}\left(\frac{\epsilon}{10^{-3}}\right)^{4}\left(\frac{v_{f . o}}{0.3}\right)^{2},
\end{aligned}
$$

where $v_{f . o}$. is the typical particle velocity at thermal freeze-out and $U_{c s} \sim \sqrt{\epsilon}$ denotes the small mixing between the $h^{s}$ and $h^{c}$ states induced by $A_{\lambda} \sim \epsilon^{2} M_{1}$. On account of this additional mixing suppression, the singlet scalar relic density is overly large. This scenario is therefore unacceptable unless higher-dimensional operators break the accidental U(1) and allow for an efficient decay of the singlet scalar [14]. 
When the gauge sector is lighter, the lightest fermion in this sector is metastable on account of $R$-parity. It is nearly degenerate with the $\mathrm{U}(1)_{x}$ gauge boson and the real scalar $h$, and thermal effects allow it to annihilate into gauge boson pairs. ${ }^{6}$ The corresponding cross-section at freeze-out is estimated to be

$$
\begin{aligned}
\langle\sigma v\rangle & \simeq \frac{g_{x}^{4} x_{H}^{4}}{16 \pi} \frac{1}{m_{Z_{x}}^{2}} v_{f . o .} \\
& \simeq\left(7 \times 10^{-24} \mathrm{~cm}^{3} / \mathrm{s}\right)\left(\frac{g_{x} x_{H}}{0.1}\right)^{4}\left(\frac{1 \mathrm{GeV}}{m_{Z_{x}}}\right)^{2}\left(\frac{v_{f . o .}}{0.3}\right),
\end{aligned}
$$

where $v_{f . o}$. denotes the typical relic velocity during freeze-out. This annihilation crosssection leads to a relic density smaller than the measured value, though such a candidate may constitute a subdominant component of the dark matter. The $h^{s}$ scalar will also be stable in the present scenario. However, it is now able to annihilate efficiently into the scalar state in the $\mathrm{U}(1)_{x}$ sector, and its corresponding relic density will be tiny.

Since we assume the gravitino is lighter than the gauge-sector LHP, the fermionic relic will decay at a later time into a gravitino and a photon. This limit was considered in [13]. The corresponding lifetime is

$$
\begin{aligned}
\tau & =\frac{16 \pi\langle F\rangle^{2}}{m_{x}^{5}\left|P_{\gamma}\right|^{2}} \\
& \simeq\left(3 \times 10^{3} s\right)\left(\frac{\sqrt{\langle F\rangle}}{100 \mathrm{TeV}}\right)^{4}\left(\frac{1 \mathrm{GeV}}{m_{x}}\right)^{5}\left(\frac{\epsilon}{\left|P_{\gamma}\right|}\right)^{2}\left(\frac{10^{-3}}{\epsilon}\right)^{2}
\end{aligned}
$$

where $P_{\gamma} \sim \epsilon$ is the projection of the light $\mathrm{U}(1)_{x}$-sector fermion onto the photino. Energetic electromagnetic decays are strongly constrained by limits on photodissociation during BBN. For the fermion relic density we estimate, ref. [74] indicates that this lifetime must be less than about $\tau \lesssim 10^{4} \mathrm{~s}$. From this, we obtain the strong upper bound on the scale of supersymmetry breaking, implying that this scenario is only viable for extremely low gauge messenger scales.

\subsection{2 $\mathrm{m}_{3 / 2} \gtrsim \mathrm{m}_{\mathrm{hid}}$}

Our findings for $m_{3 / 2} \ll m_{\text {hid }}$ lead us to consider the opposite limit, $m_{3 / 2} \gtrsim m_{\text {hid }}$. As before, we study the two cases of high-scale gauge mediation with $M_{\text {mess }} \sim 10^{14} \mathrm{GeV}$ or lower-scale gaugino mediation with a compactification scale of about the same size.

With high-scale gauge mediation without sequestering, all soft terms will receive additional supergravity contributions on the order of $m_{3 / 2}$. Assuming $m_{3 / 2} \sim \mathrm{GeV}$, the MSSM superpartner spectrum is only slightly perturbed, with any additional supergravity-induced flavor mixing being on the limit of what is consistent with current flavor bounds. The $\mathrm{U}(1)_{x}$ sector, on the other hand, is significantly modified and the precise spectrum depends on unknown UV physics. Even so, we can identify a few general features.

If the only field to condense is $H$, the theory will again split into two subsectors. The fermion mass matrix will take the same form as eq. (3.21), but now with a gaugino soft

\footnotetext{
${ }^{6}$ The radiative mass splitting is much smaller than the temperature at thermal freeze-out.
} 
mass of a similar size to the other mass terms. This will generate a gaugino-Higgsino state that is at least somewhat lighter than the gauge boson, depending on the size of the soft mass for the hidden gaugino. If the gauge sector contains the LHP, it will not have any hidden-sector annihilation modes, it will only be able to annihilate through $s$-channel $\mathrm{U}(1)_{x}$ gauge boson exchange into the visible sector. The annihilation cross-section, assuming the gravity-mediated gaugino soft mass splits the Dirac states sufficiently into two Majorana states, is

$$
\begin{aligned}
\langle\sigma v\rangle & \simeq \frac{g_{x}^{2} x_{H}^{2} e^{2} c_{W}^{2}}{12 \pi} \epsilon^{2}\left|U_{f h}\right|^{4} \frac{m_{x}^{2}}{\left(4 m_{x}^{2}-m_{Z_{x}}^{2}\right)^{2}+\Gamma_{Z_{x}}^{2} m_{Z_{x}}^{2}} v_{f . o .}^{2} \\
& \simeq\left(2 \times 10^{-29} \mathrm{~cm}^{3} / \mathrm{s}\right)\left(\frac{g_{x} x_{H}}{0.1}\right)^{2}\left(\frac{\epsilon}{10^{-3}}\right)^{2}\left|U_{f h}\right|^{4}\left(\frac{m_{x}}{1 \mathrm{GeV}}\right)^{2}\left(\frac{1 \mathrm{GeV}}{m_{Z_{x}}}\right)^{4}\left(\frac{v_{f . o .}}{0.3}\right)^{2},
\end{aligned}
$$

where $U_{f h}$ is the mixing between the Higgsino and the LHP. This cross-section is somewhat too small for the sample parameters chosen, but increases to an acceptable level for slightly lighter hidden-sector masses, a larger $\mathrm{U}(1)_{x}$ gauge coupling, or if there is a resonant enhancement of the annihilation process. Since the gravitino mass is now of the same order as the hidden-sector masses, this state will either be stable on account of $R$-parity or very long lived, and hence potentially a good dark matter candidate.

When the $\lambda$ sector is lighter and only $H$ condenses, supergravity effects make a significant contribution to the soft scalar masses for $S$ and $H^{c}$ as well as the $A_{\lambda}$ trilinear soft coupling. This mixes the $\lambda$-sector scalars, and the lighter of the two complex mass eigenstates can be heavier or lighter than the Dirac fermion in this sector. Both are stable. If the lightest scalar is the LHP, its relic abundance is given by eq. (3.28), but now with $\left|U_{c s}\right|$ typically on the order of unity due to the unsuppressed supergravity contribution to $A_{\lambda}$. This abundance can potentially be acceptable for smaller masses, larger gauge couplings, or with a resonant enhancement of the annihilation cross-section. The heavier $\lambda$-sector fermion now has its relic abundance set by annihilation to pairs of the LHP scalar with cross-section

$$
\begin{aligned}
\langle\sigma v\rangle & \simeq \frac{\lambda^{4}}{16 \pi}\left|U_{x \lambda}\right|^{4} \frac{1}{m_{x}^{2}} \\
& \simeq\left(2 \times 10^{-23} \mathrm{~cm}^{3} / \mathrm{s}\right)\left(\frac{\lambda}{0.1}\right)^{4}\left|U_{x \lambda}\right|^{4}\left(\frac{1 \mathrm{GeV}}{m_{x}}\right)^{2}
\end{aligned}
$$

where $m_{x}$ is the mass of the lightest gauge-sector fermion and $U_{x \lambda}$ its coupling to the $\lambda$-sector fermion and the LHP scalar.

If the $\lambda$-sector Dirac fermion is the LHP, its annihilation cross-section will be given by eq. (3.31), but with the replacement $m_{x} \rightarrow m_{\lambda}$ and without the $p$-wave factor of $v_{\text {f.o. }}^{2}$. This can provide an acceptable relic density for somewhat smaller masses, larger couplings, or with a resonant enhancement of the annihilation. The lightest $\lambda$-sector scalar $h_{\lambda}$ can now annihilate efficiently into the Dirac fermion LHP. The corresponding cross-section is approximately four times the value in eq. (3.32), and the resulting scalar relic density is expected to be safely small. 
If the $H, H^{c}$, and $S$ fields all condense due to additional supergravity contributions to the soft masses, the global $\mathrm{U}(1)$ in the $\lambda$ sector is broken spontaneously leading to a massless boson. This can be avoided by including a $\kappa S^{3} / 3$ coupling to the superpotential, or if there is explicit breaking by supergravity effects. The precise spectrum in this case will be UV dependent, but by analogy with the usual NMSSM scenario, it is possible to make all states in the hidden sector heavier than the $\mathrm{U}(1)_{x}$ gauge boson. In this case, the lightest stable state in the $\mathrm{U}(1)_{x}$ sector will annihilate efficiently to gauge bosons, and will typically have a safely small relic density. There is also the possibility of having a stable LHP due to $R$-parity which is mostly singlet, whose relic density can account for the observed dark matter abundance.

When supergravity effects are sequestered, as in gaugino mediation, additional contributions to the soft masses still arise from anomaly mediation, but they are suppressed by a loop factor relative to $m_{3 / 2}$. For $m_{3 / 2} \sim m_{\text {hid }}$ in this situation, we obtain an acceptable phenomenology provided $g_{x}<\lambda$. As above, this implies that the lightest $R$-odd hidden particle will be a gaugino-Higgsino mixture that is approximately degenerate with the hidden gauge boson. Since there is an approximate degeneracy, the fermion can still have phase space available to annihilate into hidden gauge bosons and obtain an acceptable relic density as in eq. 3.29, even when the cross-section for annihilation into the visible sector in eq. 3.31 is small. Since the gravitino can be heavier than the lightest fermion, there is again no problem with BBN constraints from late decays into a gravitino and a photon, and this state can again be stable and a component of the dark matter. As in the case of the bare $\mu^{\prime}$ model, we have found $m_{3 / 2} \gtrsim m_{\text {hid }}$ is desirable for a viable phenomenology.

Sequestering of supergravity effects also allows for $m_{3 / 2} \gg m_{\text {hid }}$ such that the anomalymediated soft-mass contributions are on the order of $\Delta m_{\text {hid }} \sim m_{3 / 2} g_{x}^{2} /(4 \pi)^{2}$. This is precisely the situation considered in ref. [14]. As in the case of high-scale gauge mediation with $m_{3 / 2} \sim m_{\text {hid }}$, this leads to a significant change in the $\mathrm{U}(1)_{x}$-sector spectrum (which is now largely calculable), with a similar effect. The LHP is either a Majorana Higgsinogaugino mixture, or comes from the $\lambda$ sector of the theory, and in both cases leads to too large of a relic density. This can be avoided by adding additional operators allowing for a rapid decay of this state to the visible sector, as suggested in ref. [14].

\subsection{Singlet-mediated SUSY breaking}

We now consider a scenario where a singlet field communicates between the supersymmetrybreaking sector and the $\mathrm{U}(1)_{x}$ hidden sector. This gives rise to several new features which we investigate below. The superpotential is taken to be

$$
W \supset \lambda S H H^{c}+\frac{\kappa}{3} S^{3} .
$$

The new ingredient is that we now assume that the $S$ field couples directly to the supersymmetry-breaking sector, generating a positive scalar soft mass

$$
m_{S}^{2} \simeq(100 \mathrm{GeV})^{2} .
$$

Soft terms $A_{\lambda}$ and $A_{\kappa}$ corresponding to the interaction of eq. (3.33) may also be generated, but will be suppressed if $S$ couples primarily to a $D$-term $(R$-preserving) source of 
supersymmetry breaking. The coupling of $S$ to $H$ and $H^{c}$ communicates supersymmetry breaking to the hidden sector, generating soft masses of size

$$
m_{H}^{2}=m_{H^{c}}^{2} \simeq-\frac{2 \lambda^{2}}{16 \pi^{2}} m_{S}^{2} \ln \left(\frac{\Lambda}{m_{\text {hid }}}\right)
$$

where $\Lambda$ is the scale at which the soft mass $m_{S}^{2}$ is generated. These hidden-sector soft masses are on the order of a few $\mathrm{GeV}$ provided the coupling $\lambda$ is somewhat small and the logarithm is not too large.

The resulting scalar potential is identical to that given in eq. (3.22), but with additional terms proportional to $\kappa$. With negative soft masses for $H$ and $H^{c}$, both $H$ and $H^{c}$ can develop VEVs, breaking the $\mathrm{U}(1)_{x}$ gauge symmetry and giving the $\mathrm{U}(1)_{x}$ gauge boson a mass $[6,9]$. To analyze the mass spectrum of this scenario, we make the simplifying assumptions that the kinetic mixing is suppressed, that $A_{\lambda}$ and $A_{\kappa}$ are somewhat suppressed relative to $m_{S}^{2}$, and that $\kappa \ll 1$. Under these assumptions, and assuming further that $m_{S}^{2}>0$ at the low scale, we obtain

$$
\langle H\rangle^{2} \simeq\left\langle H^{c}\right\rangle^{2} \equiv \eta^{2} \simeq-\frac{m_{H}^{2}}{\lambda^{2}},
$$

as well as

$$
\langle S\rangle \equiv s=\frac{\lambda A_{\lambda} \eta^{2}}{m_{S}^{2}} \simeq-\frac{\lambda A_{\lambda} m_{H}^{2}}{\lambda^{2} m_{S}^{2}}
$$

This minimum is stable provided $g_{x}^{4}-\left(\lambda^{2}-g_{x}^{2}\right)^{2}>0$. This yields typical values of $\eta$ in the range of $20-60 \mathrm{GeV}$ and somewhat smaller values of $s$.

The Higgs VEVs break the gauge symmetry, giving the $\mathrm{U}(1)_{x}$ gauge field a mass

$$
m_{Z_{x}}=\sqrt{2} g_{x} x_{H} \eta
$$

Expanding the scalars around their VEVs and removing the Goldstone state eaten by the $\mathrm{U}(1)_{x}$ gauge boson, $H^{(c)}=\eta+\left(h^{(c)}+i A_{H} / \sqrt{2}\right) / \sqrt{2}$ and $S=s+\left(h_{s}+i A_{S}\right) / \sqrt{2}$, the approximate mass eigenstates of the CP-even scalars are given by $h_{1} \equiv \frac{1}{\sqrt{2}}\left(h-h^{c}\right)$, $h_{2} \equiv \frac{1}{\sqrt{2}}\left(h+h^{c}\right)$, and $h_{s}=h_{s}$ with approximate masses

$$
\begin{aligned}
& m_{h_{1}}^{2} \simeq\left(4 x_{H}^{2} g_{x}^{2}-2 \lambda^{2}\right) \eta^{2} \\
& m_{h_{2}}^{2} \simeq 2 \lambda^{2} \eta^{2} \\
& m_{h_{s}}^{2} \simeq m_{S}^{2}
\end{aligned}
$$

For the CP-odd scalar masses we must take more care. In the limits of $\kappa \rightarrow 0$ or $A_{\lambda, \kappa} \rightarrow 0$, the theory has a global Abelian symmetry that is spontaneously broken by the VEVs, leading to a massless Nambu-Goldstone boson, as can be seen from the axion mass matrix in the $\left(A_{H}, A_{S}\right)$ basis

$$
\mathcal{M}_{A}^{2}=\left(\begin{array}{cc}
2 \lambda A_{\lambda} s-2 \lambda \kappa s^{2} & \sqrt{2} \lambda A_{\lambda} \eta+2 \sqrt{2} \lambda \kappa \eta s \\
\sqrt{2} \lambda A_{\lambda} \eta+2 \sqrt{2} \lambda \kappa \eta s & \lambda A_{\lambda} \frac{\eta^{2}}{s}+3 \kappa A_{\kappa} s-4 \lambda \kappa \eta^{2}
\end{array}\right) .
$$


Under our assumption that these parameters are relatively small, there remains a light pseudo-axion in the spectrum with mass

$$
m_{a_{1}}^{2} \simeq 6 \frac{s^{2}}{\eta^{2}}\left(-3 \lambda \kappa \eta^{2}+\kappa A_{\kappa} s\right) .
$$

This state derives mostly from $H$ and $H^{c}$, and the expression for its mass implies that $\lambda \kappa<0$ is needed for the stability of the perturbed minimum. The second pseudoscalar is mostly singlet and has mass $m_{a_{2}}^{2} \simeq m_{S}^{2}$.

The scalar VEVs also induce a mixing between the hidden-sector gauginos and Higgsinos. In the basis $\left(\tilde{\lambda}, \tilde{H}, \tilde{H}^{c}, \tilde{S}\right)$ the fermion mass matrix is

$$
\mathcal{M}^{f}=\left(\begin{array}{cccc}
0 & \sqrt{2} x_{H} g_{x} \eta & -\sqrt{2} x_{H} g_{x} \eta & 0 \\
\sqrt{2} x_{H} g_{x} \eta & 0 & 0 & \lambda \eta \\
-\sqrt{2} x_{H} g_{x} \eta & 0 & 0 & \lambda \eta \\
0 & \lambda \eta & \lambda \eta & 0
\end{array}\right) .
$$

This gives two fermions with mass $M_{1,2}^{f}=2 x_{H} g_{x} \eta$ and two fermions with mass $M_{3,4}^{f}=$ $\sqrt{2} \lambda \eta$.

With our simplifying assumption of small $A_{\lambda}$ and $A_{\kappa}$, the lightest state in the hidden $\mathrm{U}(1)_{x}$ sector is the pseudo-axion. In principle, these $A$-terms can be the same order as $m_{S}$ if the singlet receives its soft mass through couplings to an $R$-breaking source of supersymmetry breaking, such as the gauge messengers. On the other hand, these terms can be significantly smaller than $m_{S}^{2}$ if the dynamics generating $m_{S}^{2}$ preserves an R-symmetry. In this case the $A$-terms will be set by the dominant contribution to R-breaking in the hidden sector, which could arise from supergravity or renormalization group effects, and the lightest hidden-sector state will be the light pseudo-axion. Depending on the mass of the gravitino, the lightest $\mathrm{U}(1)_{x}$ fermion will be stable or metastable on account of $R$-parity. ${ }^{7}$ Thus, the phenomenology of the hidden sector depends on the gravitino mass, so we again consider the two cases $m_{3 / 2} \ll m_{\text {hid }}$ and $m_{3 / 2} \gtrsim m_{\text {hid }}$.

\subsection{1 $\mathrm{m}_{3 / 2} \ll \mathrm{m}_{\text {hid }}$}

With $m_{3 / 2} \ll m_{\text {hid }}$, the lightest $\mathrm{U}(1)_{x}$-sector fermion will be unstable against decaying to a pseudo-axion and a gravitino. The lifetime for this decay is

$$
\begin{aligned}
\tau & =\frac{16 \pi\langle F\rangle^{2}}{m_{\chi}^{5}\left|P_{\chi \tilde{a}}\right|^{2}} \\
& \simeq\left(3 \times 10^{-3} s\right)\left(\frac{\sqrt{\langle F\rangle}}{100 \mathrm{TeV}}\right)^{4}\left(\frac{1 \mathrm{GeV}}{m_{\chi}}\right)^{5} \frac{1}{\left|P_{\chi \tilde{a}}\right|^{2}}
\end{aligned}
$$

where $P_{\chi \tilde{a}}$ is the projection of the lightest fermion onto the superpartner of the pseudoaxion. This decay will occur safely before nucleosynthesis provided the $F$-term parameterizing supersymmetry breaking is not too large.

\footnotetext{
${ }^{7}$ Preservation of $R$-parity requires that $S, H$, and $H^{c}$ are all even.
} 
The light pseudo-axion in the hidden sector will be stable in the absence of any interactions with the SM. If these axions are heavier than an $\mathrm{eV}$ and completely stable, they create a problem with the cosmological abundance. On the other hand, they will decay away efficiently if they couple even very weakly to the SM. For example, if $S$ has a small coupling to the visible sector of the form $\zeta S H_{u} H_{d}$, the pseudo-axion will mix with the CP-odd Higgs and decay to photon pairs [75, 76]. The leading coupling in this case comes from the cross-term in the $F$-term potential due to $S$, and leads to a pseudo-axion lifetime on the order of

$$
\begin{aligned}
\tau & \simeq \frac{256 \pi^{3}}{\alpha^{2} \lambda^{2} \zeta^{2}} \frac{m_{A}^{4}}{\eta^{2} m_{a_{1}}^{3}} \\
& \simeq(0.6 s)\left(\frac{10^{-3}}{\zeta}\right)^{2}\left(\frac{0.1}{\lambda}\right)^{2}\left(\frac{m_{A}}{100 \mathrm{GeV}}\right)^{4}\left(\frac{40 \mathrm{GeV}}{\eta}\right)^{2}\left(\frac{0.1 \mathrm{GeV}}{m_{a_{1}}}\right)^{3}
\end{aligned}
$$

where $m_{A}$ is the mass of the MSSM pseudoscalar. This lifetime can thus be made safe in terms of cosmology, but is very slow relative to particle-collider timescales.

\subsection{2 $\mathrm{m}_{3 / 2} \gtrsim \mathrm{m}_{\text {hid }}$}

In the case that $m_{3 / 2} \gtrsim m_{\text {hid }}$, the lightest fermion in the $\mathrm{U}(1)_{x}$ sector will typically be the LSP, and is stable on account of $R$-parity. This state can annihilate efficiently into pseudo-axions. When the $\lambda<\sqrt{2} g_{x}$, the annihilation cross-section is on the order of

$$
\begin{aligned}
\langle\sigma v\rangle & =\frac{\lambda^{4}}{4 \pi}\left|U_{h x}\right|^{4} \frac{1}{(\sqrt{2} \lambda \eta)^{2}} \\
& \simeq\left(1 \times 10^{-23} \mathrm{~cm}^{3} / \mathrm{s}\right)\left(\frac{\lambda}{0.1}\right)^{4}\left|U_{h x}\right|^{4}\left(\frac{3 \mathrm{GeV}}{\sqrt{2} \lambda \eta}\right)^{2},
\end{aligned}
$$

where $U_{h x}$ is a gaugino-Higgsino mixing factor close to unity. We obtain a similar crosssection with $\lambda \rightarrow \sqrt{2} g_{x} x_{H}$ when $\lambda>\sqrt{2} g_{x} x_{H}$. This cross-section is large enough that the relic abundance of the stable fermion is safely small, though this state may compose a fraction of the dark matter.

\subsection{Multi-mediator models}

A minimal model with bi-fundamental mediators to a hidden $\mathrm{U}(1)_{x}$ sector consists of a pair of chiral bi-fundamentals $F$ and $F^{c}$ that transform as $Y= \pm 1 / 2$ doublets under $\mathrm{SU}(2)_{L}$ and have charges $\pm x_{F}$ under $\mathrm{U}(1)_{x} \cdot{ }^{8}$ In addition to these states, we assume that there also exists a set of fields charged only under $\mathrm{U}(1)_{x}$, such as the models described above. For simplicity, we take the superpotential for the bi-fundamentals to be

$$
W \supset \mu_{F} F F^{c}
$$

along with the soft-breaking operator

$$
V_{\text {soft }} \supset-\left[(B \mu)_{F} F F^{c}+h . c .\right] .
$$

\footnotetext{
${ }^{8}$ To preserve MSSM gauge unification, we could also incorporate $F$ and $F^{c}$ into a set of $\mathbf{5} \oplus \overline{\mathbf{5}}$ 's.
} 
While we do not specify the origin of these terms, which we assume to be of $\mathrm{TeV}$ size, they could originate dynamically from an NMSSM-like mechanism. Conversely, if the mass term $\mu_{F}$ is supersymmetric in origin, the corresponding $(B \mu)_{F}$ soft operator will arise from RG running. Pushing $\mu_{F}$ to be larger than the gauge messenger scale with $(B \mu)_{F} \rightarrow 0$, we could integrate these states out and their effect would be felt only through their contribution to kinetic mixing.

Bi-fundamental mediators can be added to any of the light $\mathrm{U}(1)_{x}$ sectors described previously. Their effect on the properties of the light sector depends importantly on the size of the $\mathrm{U}(1)_{x}$ gaugino mass they induce. As discussed in section 2.6 , if $\mu_{F}$ is a genuinely supersymmetric threshold, gaugino screening will occur and the $\mathrm{U}(1)_{x}$ gaugino mass will arise only at 5-loop order [63]. On the other hand, if the bi-fundamental threshold is not completely supersymmetric, the contribution to the $\mathrm{U}(1)_{x}$ gaugino mass will be on the order of $\left(g_{x}^{2} x_{F}^{2} /(4 \pi)^{2}\right)(B \mu)_{F} / \mu_{F}[67]$.

In the screened case, mediator effects will only significantly modify the soft masses of the light hidden-sector scalar fields. For smaller $g_{x}$, the shifts in the soft masses are subleading relative to the effect of the induced FI term, and the phenomenology of the light states will remain similar to that described above. ${ }^{9}$ In particular, the $\mu^{\prime}$ model with $\mathrm{GeV}$-scale residual supergravity contributions as well as the NMSSM scenario with a lighter gauge sector with either small $m_{3 / 2}$ or $m_{3 / 2} \sim \mathrm{GeV}$ are viable scenarios.

When the bi-fundamental mass threshold is not supersymmetric, the mediators will contribute significantly to both the gaugino and scalar soft masses in the light $\mathrm{U}(1)_{x}$ sector. Here, both the $\mu^{\prime}$ model with residual supergravity effects and the NMSSM model with small $m_{3 / 2}$ can be phenomenologically acceptable. However, the NMSSM model with larger $m_{3 / 2}$ (and only $H$ condensing) will have a problematic gaugino-Higgsino state lighter than the $\mathrm{U}(1)_{x}$ gauge boson, on account of the mediator contribution to the gaugino mass. This tends to produce too large of a relic density unless there is an enhancement of the annihilation cross-section relative to the estimate in eq. (3.31).

\section{Signatures in dark matter searches and colliders}

In this section we consider using the $\mathrm{GeV}$-scale hidden sectors studied above to help provide dark matter explanations for some of the intriguing signals seen in DAMA, PAMELA, ATIC, and PPB-BETS. We also discuss how these light hidden sectors might be probed at present and future particle-collider experiments.

\subsection{Dark matter direct detection and DAMA}

The DAMA/NaI and DAMA/LIBRA experiments, consisting of NaI-based scintillation detectors, have reported an annual modulation signal with a significance of $8.3 \sigma$ [25]. Both the period and the phase of this modulation are consistent with dark matter scattering off detector nuclei. The main challenge of such a dark matter interpretation, however, is to maintain consistency with the null results of other dark matter direct detection searches,

\footnotetext{
${ }^{9}$ With lighter bi-fundamental mediators the gauge coupling must be relatively small, $g_{x} \lesssim 0.1$, to avoid generating an unacceptably large low-scale value for the kinetic mixing $\epsilon$ through RG effects, eq. (2.6).
} 
such as CDMS [77] and XENON [78]. Two possibilities that are potentially consistent with both DAMA and other null result bounds are light $(m \lesssim 10 \mathrm{GeV})$ elastically-scattering dark matter $[26,29-31,79,80]$, and heavier $(m \gtrsim 50 \mathrm{GeV})$ inelastically-scattering dark matter $[12,32-35,81]$.

\subsubsection{Elastic dark matter}

Light elastic dark matter can produce observable recoils at DAMA, while remaining consistent with other direct detection null results. This occurs in a window where the dark matter has a mass between about 3 and $10 \mathrm{GeV}$ and a spin-independent scattering crosssection in the range $\left(10^{-41}-10^{-39}\right) \mathrm{cm}^{2}$, and depends importantly on the phenomenon of channeling $[25,26,29] .{ }^{10}$ The allowed window is constrained by the spectral shapes of the DAMA modulated and unmodulated signal rates [27, 28], but there remains an allowed region even after constraints from the spectrum of the signal are taken into account [30].

The models constructed above often contain a stable state in the multi-GeV mass range with a thermal relic density close to the measured dark matter value. Such a state could potentially act as a light elastic dark matter candidate, making up either the majority or a significant fraction of the total relic density of dark matter, provided it has an acceptable nucleon scattering cross-section. The simplest scattering mechanism in these scenarios consists of nuclear scattering mediated by the light $\mathrm{U}(1)_{x}$ gauge boson. This state can effectively mix with electric charge through kinetic mixing, and in the gauge diagonal basis the visible sector states acquire $\mathrm{U}(1)_{x}$ charges equal to $-e Q c_{W} \epsilon / g_{x}$, where $Q$ denotes the electric charge of that state. Consequently a potential dark matter state with $\mathrm{U}(1)_{x}$ charge $x_{\mathrm{DM}}$ has an effective nuclear-scattering cross-section off of a proton equal to [86]

$$
\sigma_{p} \simeq \frac{\mu_{p}^{2}}{\pi}\left(\frac{g_{x}}{M_{Z_{x}}}\right)^{4}\left(\frac{e c_{W} \epsilon}{g_{x}}\right)^{2} x_{\mathrm{DM}}^{2},
$$

where $\mu_{p} \simeq m_{p}$ is the reduced mass of the proton-DM system.

When the $\mathrm{U}(1)_{x}$ breaking is dominated by the hypercharge FI term induced by the visible-sector Higgs VEVs, we can further reduce this expression. In this case, we obtain a gauge boson mass of $m_{Z_{x}}^{2} \simeq g_{x} g_{Y}\left|c_{2 \beta} x_{H}\right| \epsilon v^{2}$. Plugging this into eq. (4.1), the factors of $\epsilon$ and $g_{x}$ amazingly cancel out, and we find

$$
\sigma_{p} \simeq\left(5 \times 10^{-38} \mathrm{~cm}^{2}\right)\left(\frac{\mu_{p}}{m_{p}}\right)^{2} \frac{1}{c_{2 \beta}^{2}}\left(\frac{x_{\mathrm{DM}}}{x_{H}}\right)^{2} .
$$

Unless there is a hierarchy in $x_{\mathrm{DM}} / x_{H}$, this cross-section is too large (assuming the local dark matter density is $0.3 \mathrm{GeV} / \mathrm{cm}^{3}$ ) by about two orders of magnitude. It can, however, be reduced if there are additional contributions to $\xi_{Y}$, or if the $\mathrm{U}(1)_{x}$ symmetry breaking is driven by other soft parameters, as in the singlet-mediated model.

A second possible scattering mechanism for a light DM candidate arises if there is a hidden-sector singlet which has a small coupling to visible-sector fields, such as through

\footnotetext{
10 There is also a spin-dependent scattering window [29, 82, 83], but it has been essentially closed by Super-Kamiokande constraints on annihilating dark matter in the sun [84, 85]. There is an exception, however, if the dark matter is not self-annihilating, in which case the constraints vanish.
} 
the terms $\lambda S H H^{c}+\zeta S H_{u} H_{d}$. Such couplings induce a small mixing among the visibleand hidden-sector Higgs states. For the models considered in sections 3.2 and 3.3, the scattering cross-section of the lightest $\mathrm{U}(1)_{x}$ sector fermion off a nucleon is approximately

$$
\begin{aligned}
& \sigma_{n} \simeq \frac{\mu_{n}^{2}}{\pi} N_{n}^{2}|U|^{4}\left(\frac{\lambda \zeta v_{u} \eta}{m_{h^{0}}^{2}}\right)^{2} \frac{1}{m_{h_{1}}^{4}} \\
& \simeq\left(2 \times 10^{-41} \mathrm{~cm}^{2}\right)\left(\frac{\mu_{n}}{m_{p}}\right)^{2}\left(\frac{N_{n}}{0.1}\right)^{2}|U|^{4}\left(\frac{\lambda}{0.1}\right)^{2}\left(\frac{\zeta}{10^{-3}}\right)^{2} \\
&\left(\frac{\eta}{20 \mathrm{GeV}}\right)^{2}\left(\frac{115 \mathrm{GeV}}{m_{h^{0}}^{2}}\right)^{4}\left(\frac{3 \mathrm{GeV}}{m_{h_{1}}}\right)^{4},
\end{aligned}
$$

where $N_{n}$ comes from the effective coupling of the exchanged scalar to the target nucleus, $U$ corresponds a mixing factor of order unity, $h^{0}$ is the MSSM Higgs, and $h_{1}$ is a hiddensector $C P$-even Higgs scalar. This cross-section is somewhat small for light elastic DM, but may be enhanced for larger values of $\zeta$ or smaller values of the hidden-sector scale.

\subsubsection{Inelastic dark matter}

A second potential dark matter explanation for the DAMA annual modulation signal is inelastic dark matter (IDM) [12, 32-35, 81]. In contrast to elastic-scattering dark matter, IDM scatters preferentially off target nuclei into a second slightly heavier state. This enhances the annual modulation of the signal and modifies the kinematics of the scattering process such that the scattering rate off heavier nuclear targets, such as the iodine in DAMA, is enhanced relative to lighter elements, such as the germanium used in CDMS. Inelastic dark matter can then account for the DAMA signal while being consistent with other direct detection bounds for a wide range of dark matter masses (above about $50 \mathrm{GeV}$ ). This requires an inelastic mass splitting on the order of $100 \mathrm{keV}$ and an effective nucleon scattering cross-section in the range $\sigma_{n} \sim\left(10^{-40}-10^{-38}\right) \mathrm{cm}^{2}$, assuming a single dominant dark matter component $[12,32-35,81]$.

IDM can arise naturally from a Dirac fermion or a complex scalar whose real components are split slightly in mass, and that couples to nuclei primarily through a massive gauge boson [32]. The couplings of the resulting mass eigenstates to the gauge boson then connect states with different masses, naturally giving rise to an inelastic interaction. Among the possibilities for the massive gauge boson mediating nuclear scattering is a light hidden $\mathrm{U}(1)_{x}$ that couples to the visible sector only through gauge kinetic mixing. The effective scattering cross-section for a dark matter particle of charge $x_{\mathrm{DM}}$ off a proton mediated by such a gauge boson was estimated in eq. (4.1). As for light elastic DM, this cross-section is slightly too large when the hidden sector symmetry breaking is dominated by the induced hypercharge FI term unless there is a hierarchy between $x_{\mathrm{DM}}$ and $x_{H}$ or if there are other contributions to the gauge boson mass. On the other hand, such a large nucleon scattering cross-section can be acceptable if the IDM makes up only a small fraction of the total dark matter density.

Coupling a TeV-scale dark matter state to a $\mathrm{GeV}$-scale hidden sector can induce an inelastic mass splitting of the right size $[8,11-14,87]$. Consider introducing a vector pair 
of chiral states $D$ and $D^{c}$ charged under $\mathrm{U}(1)_{x}$, and coupling them to the condensing Higgs $H$ in the hidden sector as well as a pair of chiral singlets $N_{1}$ and $N_{2}$,

$$
W \supset \xi_{D} N_{1} D D^{c}+\xi_{N} N_{1} N_{2}^{2}+\zeta D H N_{2} .
$$

If $N_{1} \rightarrow\left\langle N_{1}\right\rangle \sim \mathrm{TeV}$, we can integrate out the $N_{2}$ state to get the effective superpotential

$$
W_{\text {eff }} \supset-\frac{\zeta^{2}}{2 \xi_{N}\left\langle N_{1}\right\rangle}(D H)^{2} .
$$

This operator yields an inelastic mass splitting that is naturally on the order of a few hundred $\mathrm{keV}$ through the numerology $\mathrm{MeV} \sim \mathrm{GeV}^{2} / \mathrm{TeV}$. Note, however, that this operator requires $x_{H}=x_{\mathrm{DM}}$, which implies a nucleon scattering cross-section that is too large for acceptable IDM making up the full relic density when the symmetry breaking in the hidden sector is dominated by the hypercharge FI term induced by the Higgs VEVs.

Unfortunately, the minimal IDM model presented above is problematic because the heavier inelastic state tends to be very long-lived, and typically develops an unacceptably large relic density $[88,89]$. This difficulty can be avoided if the $D$ and $D^{c}$ states carry SM charges in addition to the $\mathrm{U}(1)_{x}$, as the heavier inelastic state will now be able to decay to the lighter state and neutrinos through a $Z^{0}$ gauge boson. Furthermore, the cross-section obtained from scattering through $Z^{0}$ exchange is roughly the correct size to account for the DAMA signal.

The simplest way to realize this scenario is to take $D$ and $D^{c}$ to be $\mathrm{SU}(2)$ doublets with hypercharge $Y=\mp 1 / 2$. An inelastic mass splitting can then be generated in a number of ways. Introducing a pair of states $X_{1}$ and $X_{2}$ with $\mathrm{U}(1)_{x}$ charges $\pm x_{H} / 2=\mp x_{D}$, we can write

$$
W \supset \lambda_{1} D H_{u} X_{1}+\frac{\lambda_{2}}{2} H X_{2}^{2}+M_{x} X_{1} X_{2},
$$

which, after supersymmetrically integrating out $X_{1}$ and $X_{2}$, generates the effective superpotential

$$
W_{\text {eff }} \supset \frac{\lambda_{1}^{2} \lambda_{2}}{2 M_{x}^{2}} H\left(D H_{u}\right)^{2} .
$$

This operator can generate the correct inelastic splitting for $\lambda_{1} \sim \lambda_{2} \sim 0.1$ and $M_{x} \sim \mathrm{TeV}$ when $\langle H\rangle \sim \mathrm{GeV}$.

A second related scenario that is able to induce the correct inelastic splitting consists of a singlet $S$ in addition to $X_{1}$ and $X_{2}$, and the superpotential

$$
W \supset \lambda_{1} D H_{u} X_{1}+\lambda_{2} H X_{2} S+\frac{1}{2} M_{s} S^{2}+M_{x} X_{1} X_{2},
$$

where $X_{1}$ and $X_{2}$ now have $\mathrm{U}(1)_{x}$ charges $\pm x_{H}$. Integrating out $N, X_{1}$, and $X_{2}$ at the supersymmetric level then generates the inelastic mass-splitting operator

$$
W_{\text {eff }} \supset-\frac{\lambda_{1}^{2} \lambda_{2}^{2}}{2 M_{s} M_{x}^{2}}\left(D H_{u} H\right)^{2} .
$$


In this case, we obtain an inelastic mass splitting on the order of $100 \mathrm{keV}$ for $M_{x} \sim M_{s} \sim$ $300 \mathrm{GeV},\langle H\rangle \sim 2 \mathrm{GeV}$, and $\lambda_{1} \sim \lambda_{2} \sim 0.5$.

The two IDM scenarios with bi-fundamental $D$ and $D^{c}$ fields described above are very similar to the multi-mediator models discussed in section 3.4. We found that these models can lead to a phenomenologically acceptable $\mathrm{GeV}$-scale $\mathrm{U}(1)_{x}$ sector in a number of ways. Scattering of the bi-fundamental IDM off nuclei will be mediated both by the SM $Z^{0}$ as well as the light $\mathrm{U}(1)_{x}$ gauge boson (provided there is kinetic mixing). Somewhat amusingly, the scattering of this IDM state off protons will be dominated by the $\mathrm{U}(1)_{x}$ gauge boson exchange, while its scattering off neutrons will be dominated by the SM $Z^{0}$. The relic density of the IDM will be determined in a large part by its annihilation to gauge bosons. For fermionic $\mathrm{SU}(2)_{L}$-doublet IDM, this implies that the mass of the state must be greater than about $1000 \mathrm{GeV}$ to provide the observed relic density, while for scalars the mass should be in excess of about $500 \mathrm{GeV}$ [90]. However, lighter IDM that makes up only a small fraction of the total dark matter relic density can still potentially account for the DAMA signal owing to the often large proton scattering cross-section mediated by the $\mathrm{U}(1)_{x}$ gauge boson.

\subsection{Applications to PAMELA and ATIC}

The PAMELA [18], ATIC [19], and PPB-BETS [20] experiments observe excesses in cosmic ray positrons and electrons at energies above $10 \mathrm{GeV}$. These signals could potentially originate from dark matter annihilating in our galaxy. For such an explanation to work, the dark matter state must be heavier than about $100 \mathrm{GeV}$ and annihilate efficiently into leptons with a cross-section larger than the value providing the correct thermal relic density [24].

The light $\mathrm{U}(1)_{x}$ models outlined above, when coupled to a heavier dark matter state charged under the $\mathrm{U}(1)_{x}$, can have the correct properties to induce the necessary enhancement in a subset of the phenomenologically consistent parameter space [8]. Annihilation of the heavy $\mathrm{U}(1)_{x}$-charged dark matter state into $\mathrm{U}(1)_{x}$ gauge bosons in our local region of the galaxy will generically receive a Sommerfeld enhancement provided $\alpha_{x} m_{\mathrm{DM}} / m_{Z_{x}} \gtrsim 1$. To account for the signals at PAMELA or ATIC without violating observational constraints on fluxes of gamma rays [91-94] and anti-protons [24], these dark gauge bosons should subsequently decay primarily to leptons. This occurs automatically due to kinematics for $m_{Z_{x}} \lesssim 0.3 \mathrm{GeV}$ provided the $\mathrm{U}(1)_{x}$ gauge boson is also lighter than twice the mass of any of the other hidden-sector states.

\subsection{Collider phenomenology}

The presence of supersymmetric hidden sectors at the $\mathrm{GeV}$ scale can lead to a variety of interesting signals at particle colliders. First, at very high-energy colliders such as the Tevatron and the LHC, the visible-sector LSP produced in cascade decays is unstable against subsequently decaying into hidden-sector states, as discussed in ref. [95], and more recently in refs. $[6,8,9,11]$. The hidden-sector particles may then cascade further, potentially giving rise to additional visible- and hidden-sector final states. Second, at high luminosity $e^{+} e^{-}$machines, such as the $\mathrm{B}$ and charm factories, heavy-flavor mesons will 
have rare exotic decays into the hidden sector, such as $e^{+} e^{-} \rightarrow \Upsilon \rightarrow \gamma+$ hidden, and $e^{+} e^{-} \rightarrow \gamma+$ hidden through an ISR photon. For the models discussed in the previous sections, we find that their collider signatures involve photons plus missing energy, and in some cases highly collimated "lepton jets" $[8,11]$. We give a brief overview of potential collider signatures here, leaving a more detailed study for future work.

\subsubsection{High-energy hadron colliders}

We consider first some of the potential signatures of an Abelian hidden sector at highenergy hadron colliders such as the Tevatron and the LHC. The production of hiddensector particles will arise primarily through the cascade decays of heavier states carrying SM charges. In particular, the visible-sector LSP will itself be produced through cascade decays in the usual way, and may subsequently decay into the lighter hidden sector. Hiddensector states can also be produced through the decays of (necessarily) heavy bi-fundamental states charged under both the SM and $\mathrm{U}(1)_{x}$ gauge groups. This is a very simple example of a hidden valley scenario [96]. For both cases, additional visible-sector states may also be emitted in the LSP or bi-fundamental decay.

The subsequent cascade in the hidden sector can be a source of further visible-sector particles. The general condition for this is that $\mathrm{U}(1)_{x}$ gauge boson decay predominantly into the visible sector, which usually requires that it is kinematically incapable of decaying to pairs of hidden-sector particles. Other hidden-sector states may also have decay modes to the visible sector, but they are generally very slow on collider time scales in the scenarios considered above. When the $\mathrm{U}(1)_{x}$ gauge boson decays primarily to hidden states, the hidden-sector cascades will unfortunately remain hidden.

Consider the case where the visible-sector LSP (vLSP) is a neutralino, and the U(1) $x$ gauge boson decays mainly back to visible states. The decay chains with a squark or slepton vLSP will be similar, but with one more quark or lepton. The dominant decay mode of the neutralino is $\chi^{0} \rightarrow H \tilde{H}$, where $H$ and $\tilde{H}$ are hidden Higgs and Higgsino fields [11]. These states then cascade down to the lightest fermion and scalar fields in the hidden sector allowed by phase space. Along the way, one or more $\mathrm{U}(1)_{x}$ gauge bosons can be emitted. These will typically decay promptly to highly collimated leptons (and possibly pions), giving rise to low-invariant mass "lepton jets" [8, 11, 97, 98]. In the models we have considered, both the final state fermions and scalars (including the R-even states) are long-lived on collider scales and leave the detector (see eqs. (3.17), (3.47)). This necessarily gives rise to a large component of missing energy accompanying the "lepton jet". Note, however, that the non-Abelian models considered in $[8,98]$ have characteristically busy events due to showering in the hidden sector and the final-state leptons will tend to be somewhat soft. On the other hand, the Abelian models have comparatively quiet decay chains in the hidden sector and the leptons will thus carry a higher fraction of the total momentum of the event.

An alternative possibility is that the connection between the hidden sector and visible sector is via a singlet which has a small coupling with the visible-sector Higgs fields and a large coupling to hidden-sector Higgs fields. For example, we can consider the model of section 3.3 with the superpotential coupling $W \supset \zeta S H_{u} H_{d}$ and $\zeta \ll 1$. This coupling is 
not necessary to communicate SUSY breaking, but it is one possible way to ensure that the lightest axion has a decay mode. In this case, a small component of the neutralino is the singlino, which will again lead to the decay $\chi^{0} \rightarrow H \tilde{H}$ and similar signatures as before.

\subsubsection{Lower-energy $e^{+} e^{-}$colliders}

Lower-energy $e^{+} e^{-}$machines, such as Belle, BaBar, DA $\Phi$ NE, KLOE and CLEO, offer highluminosity precision tests of these low-mass hidden sectors by production of the hiddensector particles through mixing of the $\mathrm{U}(1)_{x}$ gauge boson with the photon. Potentially interesting processes include $e^{+} e^{-} \rightarrow \gamma+$ hidden where the hidden states have invisible decay modes and the photon arises from ISR, $e^{+} e^{-} \rightarrow$ hidden with subsequent decays of the hidden particles producing SM final states, as well as hidden decays of SM resonances, such as the $\Upsilon(1 S)$. We discuss signatures in both gauge- and singlet-mediated models, leaving more detailed studies for future work. For related recent studies of hidden-sector $e^{+} e^{-}$collider signatures, see refs. [99-103].

When there is gauge kinetic mixing of the $\mathrm{U}(1)_{x}$ with electric charge, hidden-sector states are produced by the $s$-channel exchange of the $\mathrm{U}(1)_{x}$ gauge boson, $e^{+} e^{-} \rightarrow X \bar{X}$, or through a $t$-channel electron exchange, $e^{+}+e^{-} \rightarrow \gamma Z_{x}$. The related production crosssections are given in refs. [99, 100], which find that for $\mathrm{GeV}$-scale mediators and a kinetic mixing of $\epsilon \sim 10^{-3}$, production cross-sections at low-energy $e^{+} e^{-}$machines are typically in the $\mathrm{fb}$ range. Given the $\mathrm{ab}^{-1}$ collected at the $B$ factories, this implies hundreds to thousands of hidden-sector particles have been produced at these machines. Search modes, and the corresponding constraints from existing searches, then depend on the decay chains of the hidden particles $[99,100]$. The simplest signatures result when the hidden states remain stable on collider timescales, so that the search is simply for a photon plus missing energy. This type of general search remains to be done. As in the previous discussion on hadron-collider signatures, in the cascade decays of hidden Higgs and Higgsinos, $Z_{x}$ gauge bosons may be radiated which decay to pairs of leptons with low invariant mass.

The Belle and CLEO collaborations have already put a strong constraint on these sectors by searching for $\Upsilon(3 S, 2 S) \rightarrow \Upsilon(1 S)+\gamma \rightarrow$ hidden $+\gamma$ [104, 105], where the $\Upsilon(1 S)$ decays to hidden particles via photon mixing. Belle finds the stronger constraint at $B(\Upsilon(1 S) \rightarrow$ invisible $)<2.5 \times 10^{-3}$, which should be satisfied as long as $\epsilon \lesssim 10^{-2-3}$. These bounds apply for both direct decays to the LHP, and heavier hidden-sector states which are either meta-stable on collider time scales or decay to states which are meta-stable.

For the singlet-mediated models, any production of hidden-sector particles must go through mixing of visible and hidden Higgses. Because the coupling of the Higgs to the initial state is very weak, constraints from $e^{+} e^{-}$colliders are also typically very weak. A possible exception is through the exotic decay $\Upsilon \rightarrow \gamma+$ hidden, as discussed in [106]. The $\Upsilon$ can decay to an (off-shell) MSSM pseudoscalar Higgs and photon, with the MSSM pseudoscalar Higgs mixing with a hidden Higgs, $h^{c}$ or $h$. The hidden Higgs may then decay to two LHPs, resulting in a completely invisible decay of the hidden Higgs. Since $\zeta \ll 1$, however, these constraints also turn out to be quite weak. 


\section{Conclusions}

As long as there is an asymmetry in the way that various particles feel supersymmetry breaking, it is generic to end up with sectors at hierarchically different mass scales. This seems particularly likely when supersymmetry breaking is communicated to the visible sector through gauge interactions, in which case particles that are not charged under the messenger gauge group will receive suppressed contributions to their soft masses. If there are no additional couplings between these particles and the visible sector, one generically expects the mass scale of the hidden sector to be set by the gravitino mass. On the other hand, it is easy for additional mediator fields (e.g., high-scale fields charged under both visible and hidden gauge groups) to feed supersymmetry breaking into the hidden sector through loop-suppressed contributions, giving rise to $\mathrm{GeV}$-scale hidden sectors.

Perhaps one of the simplest ways to add a $\mathrm{GeV}$-scale hidden sector is to consider a new $\mathrm{U}(1)_{x}$ gauge group and a vector-like pair of fields charged under it. If the $\mathrm{U}(1)_{x}$ couples to the visible sector through kinetic mixing with hypercharge, SUSY breaking is communicated to the hidden sector through the kinetic mixing. Even in the absence of kinetic mixing, viable scenarios can arise when a singlet couples to both the SUSY-breaking and hidden sectors, but with a suppressed coupling to the hidden sector.

Some care must be taken in the construction of these models, however. For example, if the gravitino is lighter than the states in the hidden sector, there is always a danger of quasi-stable states which either decay after BBN or have too large of a relic density. In this case, viable scenarios arise only for a very low SUSY-breaking scale or if higherdimensional operators allow for additional decays. This situation is generally alleviated if the gravitino is heavier than the lightest hidden-sector particle, provided it retains a large enough annihilation channel so that its relic abundance isn't too large. This light stable particle is typically a good candidate for the dark matter, though its mass is much less than the weak scale. A particularly interesting scenario arises when supergravity effects are sequestered and $m_{3 / 2} \sim m_{\text {hid }}$ - the lightest fermion is kinematically not allowed to decay to a gravitino and photon, but can still annihilate to hidden-sector states which then decay. This scenario is viable when the supersymmetry breaking is communicated to the hidden sector either via kinetic mixing or via a singlet.

New particles and forces at the GeV scale may also be relevant for explaining some of the recent possible hints for dark matter seen by the DAMA, PAMELA, ATIC, and PPB-BETS experiments. The annual modulation signal seen at DAMA can potentially be explained by the elastic scattering of a $\mathrm{GeV}$-scale component of dark matter, or by heavier dark matter scattering inelastically to a state that is heavier by $\sim 100 \mathrm{keV}$, with the new sector at the $\mathrm{GeV}$ scale naturally inducing the splitting. GeV-scale gauge bosons can also give rise to a Sommerfeld enhancement of the dark matter annihilation today, giving a possible explanation for the excess in electrons and positrons reported by PAMELA, ATIC, and PPB-BETS. In addition, these $\mathrm{GeV}$-scale hidden sectors can potentially be probed at both future and present hadron and $e^{+} e^{-}$colliders.

Whether these sectors are responsible for the signals observed by the recent results from the dark matter experiments, the presence of such sectors can be quite generic, and 
the dynamics and signatures very different from MSSM phenomenology. Dark matter experiments, as well $e^{+} e^{-}$and hadron colliders, can then give us many possible windows into the hidden world.

\section{Acknowledgments}

We thank Brian Batell, Doug Finkbeiner, Jonathan Heckman, Dan Hooper, Markus Luty, John Mason, Peter Ouyang, Frank Petriello, Matt Schwartz, Tracy Slatyer, and Tomer Volansky for helpful discussions. This work is supported in part by the Harvard Center for the Fundamental Laws of Nature, by NSF grant PHY-0556111 (DP), and by the DOE including grant DE-FG02-95ER40896 (KMZ).

\section{A Kinetic mixing mediation}

Supersymmetry breaking in the visible (MSSM) sector can be mediated to an Abelian $\mathrm{U}(1)_{x}$ sector by gauge kinetic mixing. In order to study these effects, we use the method of analytic continuation into superspace [62,63]. For this, we determine the dependence of the running hidden-sector gauge couplings $g_{a}(\mu)$ and wavefunction factors $Z_{i}(\mu)$ on the gauge messenger mass scale $M$, and then promote $M$ to a chiral superfield $X$ or a real superfield $\sqrt{X^{\dagger} X}$. The result of this procedure represents the leading term in an expansion in $\left|F / M^{2}\right|$ of the soft terms, with the full result obtainable from a diagrammatic analysis [107].

It is convenient to work in the holomorphic basis where the gauge couplings appear in front of the gauge kinetic terms and are promoted to chiral superfields. In this basis, the effective Lagrangian at scale $\mu$ takes the form

$$
\begin{aligned}
\mathcal{L}= & \int d^{2} \theta\left[\frac{1}{4 g_{Y}^{2}(\mu)} B^{\alpha} B_{\alpha}+\frac{1}{4 g_{x}^{2}(\mu)} X^{\alpha} X_{\alpha}+\frac{\epsilon_{h}(\mu)}{2} B^{\alpha} X_{\alpha}\right]+\text { h.c. } \\
& +\int d^{4} \theta\left[Z_{i}(\mu) \phi_{i}^{\dagger} e^{x_{i} V_{x}} \phi_{i}+\ldots\right]
\end{aligned}
$$

Note that $\epsilon_{h}$ is related to the kinetic mixing in the canonically normalized basis at leading order as

$$
\epsilon(\mu)=\epsilon_{h}(\mu) g_{Y}(\mu) g_{x}(\mu)
$$

The exact RG equations for the holomorphic gauge couplings are

$$
\frac{d}{d t}\left(\frac{1}{g_{a}^{2}}\right)=\frac{b_{a}}{8 \pi^{2}}, \quad a=x, Y,
$$

where $b_{a}=-\sum_{i} q_{i}^{a} q_{i}^{a}$ is the beta function coefficient (and $q_{i}^{a}$ denotes the charge of field $i$ ). The holomorphic-basis kinetic mixing runs according to

$$
\frac{d}{d t} \epsilon_{h}=\frac{b_{x Y}}{8 \pi^{2}}
$$

with $b_{x Y}=-\sum_{i} x_{i} Y_{i}$. From this, we see that: if there are no fields charged under both $\mathrm{U}(1)_{x}$ and $\mathrm{U}(1)_{Y}$, the holomorphic kinetic mixing does not run at all. Upon transforming 
to the canonical basis with the kinetic mixing eliminated, we reproduce the RG equations listed in ref. [53].

Let us assume the gauge messengers are charged only under the SM gauge groups, and that there are no fields at the messenger scale charged under both hypercharge and the $\mathrm{U}(1)_{x}$ group. In this case, only $b_{Y}$ (but not $b_{x}$ or $\epsilon_{h}$ ) changes across the messenger threshold, only $g_{Y}(\mu)$ depends on the messenger mass, and only a $\mathrm{U}(1)_{Y}$ gaugino mass is generated in this basis. The gaugino mass matrix in the basis with explicit kinetic mixing is then simply

$$
M_{\text {gaugino }}=\left(\begin{array}{cc}
M_{1} & 0 \\
0 & 0
\end{array}\right) .
$$

The physical gaugino masses can receive higher loop corrections through the messenger mass dependence of the wavefunction renormalization $Z_{i}$, since it contributes to the physical gauge couplings through the rescaling anomaly associated with going into a canonical basis for the kinetic terms. This correction to $M_{x}$ is tiny, however, and will be of order $\sim \frac{\epsilon_{h}^{2}}{\left(16 \pi^{2}\right)^{3}} \frac{F}{M}$.

We turn next to the scalar masses in the light hidden sector. The one-loop anomalous dimension of a hidden field $\phi_{i}$ with $\mathrm{U}(1)_{x}$ charge $x_{i}$ is given by

$$
\begin{aligned}
\frac{d \ln Z_{i}}{d t} & =\frac{x_{i}^{2}}{4 \pi^{2}} \frac{g_{x}^{2}}{1-g_{x}^{2} g_{Y}^{2} \epsilon_{h}^{2}} \\
& \simeq \frac{x_{i}^{2}}{4 \pi^{2}}\left[g_{x}^{2}(\mu)+\epsilon_{h}^{2} g_{Y}^{2}(\mu, M) g_{x}^{4}(\mu)+O\left(\epsilon_{h}^{4}\right)\right] .
\end{aligned}
$$

This can be obtained by resumming double insertions of $\epsilon_{h}$ on gauge boson propagators in the mixed kinetic basis, or by transforming to a basis with canonical kinetic terms and no explicit kinetic mixing. Only the second term in the last line of eq. (A.7) depends on the messenger mass, so we see immediately that the squared scalar masses are suppressed by $\epsilon_{h}^{2}$. Since this contribution to the anomalous dimension is proportional to that of the right-handed selectron (normalized to have hypercharge $Y=1$ ), we obtain

$$
\left.\frac{\partial^{2} \ln Z_{i}}{\partial M^{2}}\right|_{\mu=M}=\left.\epsilon_{h}^{2} x_{i}^{2} g_{x}^{4}(M) \frac{\partial^{2} \ln Z_{E^{c}}}{\partial M^{2}}\right|_{\mu=M} .
$$

From this we can simply read off the soft masses generated at the messenger scale:

$$
m_{i}^{2}(M)=\epsilon_{h}^{2} x_{i}^{2} g_{x}^{4}(M) m_{E^{c}}^{2}(M)=\epsilon^{2}(M) \frac{x_{i}^{2} g_{x}^{2}(M)}{g_{Y}^{2}(M)} m_{E^{c}}^{2}(M) .
$$

We stress that these results are quite general, in that they do not depend on the details of the messenger sector. Furthermore, this result can also be derived by considering the two-loop graph for the scalar $\phi_{i}$ communicating to the messengers through the kinetic mixing term.

Finally, as in gauge mediation to the visible sector, hidden-sector A-terms and B-terms can be generated at the two-loop level in the presence of hidden-sector trilinear or bilinear couplings. These terms will be generated at the messenger scale at order $\sim \frac{\epsilon^{2}}{\left(16 \pi^{2}\right)^{2}} \frac{F}{M}$, and will be subdominant relative to the RG effects discussed in section 2.5. 


\section{B Renormalization group equations}

We collect here the one-loop renormalization group equations for the soft terms in the models considered in the text. Throughout, we implicitly shift the visible- and hiddensector scalar soft masses by explicit FI terms such that $\operatorname{Tr}\left(Y m^{2}\right)=\operatorname{Tr}\left(x m^{2}\right)=0$ [108].

\section{B.1 Minimal $\mu^{\prime}$ model}

The only hidden-sector interactions within this model are the $\mathrm{U}(1)_{x}$ gauge interactions. This leads to the RG equations

$$
\begin{aligned}
& (4 \pi)^{2} \frac{d m_{H^{(c)}}^{2}}{d t}=-8 s_{\epsilon}^{2} x_{H}^{2} g_{x}^{2}\left|M_{1}\right|^{2} \\
& (4 \pi)^{2} \frac{d\left(B \mu^{\prime}\right)}{d t}=-4 x_{H}^{2} g_{x}^{2}\left(B \mu^{\prime}\right)-8 s_{\epsilon}^{2} x_{H}^{2} g_{x}^{2}\left(M_{1} \mu^{\prime}\right) .
\end{aligned}
$$

\section{B.2 Hidden sector NMSSM}

In addition to gauge interactions, there is now a Yukawa interaction with coupling $\lambda$ (and potentially a singlet self-coupling $\kappa$ ). This leads to the RG equations

$$
\begin{aligned}
(4 \pi)^{2} \frac{d \ln \lambda}{d t} & =3|\lambda|^{2}+2|\kappa|^{2}-4 x_{H}^{2} g_{x}^{2} \\
(4 \pi)^{2} \frac{d \ln \kappa}{d t} & =3|\lambda|^{2}+6|\kappa|^{2} \\
(4 \pi)^{2} \frac{d m_{H^{(c)}}^{2}}{d t} & =2|\lambda|^{2}\left(m_{H}^{2}+m_{H^{c}}^{2}+m_{S}^{2}+\left|A_{\lambda}\right|^{2}\right)-8 s_{\epsilon}^{2} x_{H}^{2} g_{x}^{2}\left|M_{1}\right|^{2} \\
(4 \pi)^{2} \frac{d m_{S}^{2}}{d t} & =2|\lambda|^{2}\left(m_{H}^{2}+m_{H^{c}}^{2}+m_{S}^{2}+\left|A_{\lambda}\right|^{2}\right)+4|\kappa|^{2}\left(3 m_{s}^{2}+\left|A_{\kappa}\right|^{2}\right) \\
(4 \pi)^{2} \frac{d A_{\lambda}}{d t} & =6|\lambda|^{2} A_{\lambda}+4|\kappa|^{2} A_{\kappa}-8 s_{\epsilon}^{2} x_{H}^{2} g_{x}^{2} M_{1} \\
(4 \pi)^{2} \frac{d A_{\kappa}}{d t} & =12|\kappa|^{2} A_{\kappa}+6|\lambda|^{2} A_{\lambda} .
\end{aligned}
$$

\section{References}

[1] For reviews, see S.P. Martin, A supersymmetry primer, hep-ph/9709356 [SPIRES]; D.J.H. Chung et al., The soft supersymmetry-breaking lagrangian: theory and applications, Phys. Rept. 407 (2005) 1 [hep-ph/0312378] [SPIRES].

[2] A.H. Chamseddine, R.L. Arnowitt and P. Nath, Locally supersymmetric grand unification, Phys. Rev. Lett. 49 (1982) 970 [SPIRES];

R. Barbieri, S. Ferrara and C.A. Savoy, Gauge models with spontaneously broken local supersymmetry, Phys. Lett. B 119 (1982) 343 [SPIRES];

L.J. Hall, J.D. Lykken and S. Weinberg, Supergravity as the messenger of supersymmetry breaking, Phys. Rev. D 27 (1983) 2359 [SPIRES];

N. Ohta, Grand unified theories based on local supersymmetry,

Prog. Theor. Phys. 70 (1983) 542 [SPIRES].

[3] H.P. Nilles, Supersymmetry, supergravity and particle physics, Phys. Rept. 110 (1984) 1 [SPIRES]. 
[4] M. Dine and W. Fischler, A phenomenological model of particle physics based on supersymmetry, Phys. Lett. B 110 (1982) 227 [SPIRES]; A supersymmetric GUT, Nucl. Phys. B 204 (1982) 346 [SPIRES];

L. Álvarez-Gaumé, M. Claudson and M.B. Wise, Low-energy supersymmetry, Nucl. Phys. B 207 (1982) 96 [SPIRES];

S. Dimopoulos and S. Raby, Geometric hierarchy, Nucl. Phys. B 219 (1983) 479 [SPIRES].

[5] M. Dine, A.E. Nelson and Y. Shirman, Low-energy dynamical supersymmetry breaking simplified, Phys. Rev. D 51 (1995) 1362 [hep-ph/9408384] [SPIRES];

M. Dine, A.E. Nelson, Y. Nir and Y. Shirman, New tools for low-energy dynamical supersymmetry breaking, Phys. Rev. D 53 (1996) 2658 [hep-ph/9507378] [SPIRES].

[6] D. Hooper and K.M. Zurek, Natural supersymmetric model with MeV dark matter, Phys. Rev. D 77 (2008) 087302 [arXiv:0801.3686] [SPIRES].

[7] J.L. Feng and J. Kumar, The WIMPless miracle: dark-matter particles without weak-scale masses or weak interactions, Phys. Rev. Lett. 101 (2008) 231301 [arXiv:0803.4196] [SPIRES].

[8] N. Arkani-Hamed, D.P. Finkbeiner, T.R. Slatyer and N. Weiner, A theory of dark matter, Phys. Rev. D 79 (2009) 015014 [arXiv:0810.0713] [SPIRES];

N. Arkani-Hamed and N. Weiner, LHC signals for a superunified theory of dark matter, JHEP 12 (2008) 104 [arXiv:0810.0714] [SPIRES].

[9] K.M. Zurek, Multi-component dark matter, Phys. Rev. D 79 (2009) 115002 [arXiv:0811.4429] [SPIRES].

[10] E.J. Chun and J.-C. Park, Dark matter and sub-GeV hidden U(1) in GMSB models, JCAP 02 (2009) 026 [arXiv: 0812.0308] [SPIRES].

[11] M. Baumgart, C. Cheung, J.T. Ruderman, L.-T. Wang and I. Yavin, Non-abelian dark sectors and their collider signatures, JHEP 04 (2009) 014 [arXiv: 0901.0283] [SPIRES].

[12] Y. Cui, D.E. Morrissey, D. Poland and L. Randall, Candidates for inelastic dark matter, JHEP 05 (2009) 076 [arXiv:0901.0557] [SPIRES].

[13] C. Cheung, J.T. Ruderman, L.-T. Wang and I. Yavin, Kinetic mixing as the origin of light dark scales, arXiv:0902.3246 [SPIRES].

[14] A. Katz and R. Sundrum, Breaking the dark force, JHEP 06 (2009) 003 [arXiv: 0902 . 3271] [SPIRES].

[15] L. Randall and R. Sundrum, Out of this world supersymmetry breaking, Nucl. Phys. B 557 (1999) 79 [hep-th/9810155] [SPIRES].

[16] G.F. Giudice, M.A. Luty, H. Murayama and R. Rattazzi, Gaugino mass without singlets, JHEP 12 (1998) 027 [hep-ph/9810442] [SPIRES].

[17] M. Pospelov, Secluded U(1) below the weak scale, arXiv:0811.1030 [SPIRES];

M. Pospelov and A. Ritz, Astrophysical signatures of secluded dark matter, Phys. Lett. B 671 (2009) 391 [arXiv:0810.1502] [SPIRES].

[18] PAMELA collaboration, O. Adriani et al., An anomalous positron abundance in cosmic rays with energies 1.5.100 GeV, Nature 458 (2009) 607 [arXiv:0810.4995] [SPIRES].

[19] J. Chang et al., An excess of cosmic ray electrons at energies of $300.800 \mathrm{GeV}$, Nature 456 (2008) 362 [SPIRES]. 
[20] PPB-BETS collaboration, S. Torii et al., High-energy electron observations by PPB-BETS flight in Antarctica, arXiv:0809.0760 [SPIRES].

[21] D.P. Finkbeiner, Microwave ISM emission observed by WMAP, Astrophys. J. 614 (2004) 186 [astro-ph/0311547] [SPIRES].

[22] G. Dobler and D.P. Finkbeiner, Extended anomalous foreground emission in the WMAP 3-year data, Astrophys. J. 680 (2008) 1222 [arXiv:0712.1038] [SPIRES].

[23] D. Hooper, D.P. Finkbeiner and G. Dobler, Evidence of dark matter annihilations in the WMAP haze, Phys. Rev. D 76 (2007) 083012 [arXiv:0705.3655] [SPIRES].

[24] M. Cirelli, M. Kadastik, M. Raidal and A. Strumia, Model-independent implications of the $e^{+}, e^{-}$, anti-proton cosmic ray spectra on properties of dark matter,

Nucl. Phys. B 813 (2009) 1 [arXiv:0809.2409] [SPIRES].

[25] DAMA collaboration, R. Bernabei et al., First results from DAMA/LIBRA and the combined results with DAMA/NaI, Eur. Phys. J. C 56 (2008) 333 [arXiv:0804.2741] [SPIRES].

[26] F. Petriello and K.M. Zurek, DAMA and WIMP dark matter, JHEP 09 (2008) 047 [arXiv:0806.3989] [SPIRES].

[27] S. Chang, A. Pierce and N. Weiner, Using the energy spectrum at DAMA/LIBRA to probe light dark matter, arXiv:0808.0196 [SPIRES].

[28] M. Fairbairn and T. Schwetz, Spin-independent elastic WIMP scattering and the DAMA annual modulation signal, JCAP 01 (2009) 037 [arXiv:0808.0704] [SPIRES].

[29] C. Savage, G. Gelmini, P. Gondolo and K. Freese, Compatibility of DAMA/LIBRA dark matter detection with other searches, JCAP 04 (2009) 010 [arXiv:0808.3607] [SPIRES].

[30] C. Savage, K. Freese, P. Gondolo and D. Spolyar, Compatibility of DAMA/LIBRA dark matter detection with other searches in light of new galactic rotation velocity measurements, arXiv:0901.2713 [SPIRES].

[31] G. Gelmini and P. Gondolo, DAMA dark matter detection compatible with other searches, hep-ph/0405278 [SPIRES];

P. Gondolo and G. Gelmini, Compatibility of DAMA dark matter detection with other searches, Phys. Rev. D 71 (2005) 123520 [hep-ph/0504010] [SPIRES].

[32] D. Tucker-Smith and N. Weiner, Inelastic dark matter, Phys. Rev. D 64 (2001) 043502 [hep-ph/0101138] [SPIRES].

[33] D. Tucker-Smith and N. Weiner, Inelastic dark matter at DAMA, CDMS and future experiments, Nucl. Phys. Proc. Suppl. 124 (2003) 197 [astro-ph/0208403] [SPIRES].

[34] D. Tucker-Smith and N. Weiner, The status of inelastic dark matter, Phys. Rev. D 72 (2005) 063509 [hep-ph/0402065] [SPIRES].

[35] S. Chang, G.D. Kribs, D. Tucker-Smith and N. Weiner, Inelastic dark matter in light of DAMA/LIBRA, Phys. Rev. D 79 (2009) 043513 [arXiv:0807.2250] [SPIRES].

[36] G. Weidenspointner et al., The sky distribution of positronium annihilation continuum emission measured with SPI/INTEGRAL, astro-ph/0601673 [SPIRES].

[37] C. Boehm and P. Fayet, Scalar dark matter candidates, Nucl. Phys. B 683 (2004) 219 [hep-ph/0305261] [SPIRES]; 
C. Boehm, P. Fayet and J. Silk, Light and heavy dark matter particles,

Phys. Rev. D 69 (2004) 101302 [hep-ph/0311143] [SPIRES];

C. Bouchiat and P. Fayet, Constraints on the parity-violating couplings of a new gauge boson, Phys. Lett. B 608 (2005) 87 [hep-ph/0410260] [SPIRES].

[38] M. Pospelov, A. Ritz and M.B. Voloshin, Secluded WIMP dark matter, Phys. Lett. B 662 (2008) 53 [arXiv:0711.4866] [SPIRES].

[39] P. Fayet, Light spin-1/2 or spin-0 dark matter particles, Phys. Rev. D 70 (2004) 023514 [hep-ph/0403226] [SPIRES].

[40] D.E. Kaplan, G.D. Kribs and M. Schmaltz, Supersymmetry breaking through transparent extra dimensions, Phys. Rev. D 62 (2000) 035010 [hep-ph/9911293] [SPIRES].

[41] Z. Chacko, M.A. Luty, A.E. Nelson and E. Ponton, Gaugino mediated supersymmetry breaking, JHEP 01 (2000) 003 [hep-ph/9911323] [SPIRES].

[42] M.A. Luty and R. Sundrum, Supersymmetry breaking and composite extra dimensions, Phys. Rev. D 65 (2002) 066004 [hep-th/0105137] [SPIRES]; Anomaly mediated supersymmetry breaking in four dimensions, naturally, Phys. Rev. D 67 (2003) 045007 [hep-th/0111231] [SPIRES].

[43] M. Schmaltz and R. Sundrum, Conformal sequestering simplified, JHEP 11 (2006) 011 [hep-th/0608051] [SPIRES].

[44] T.S. Roy and M. Schmaltz, A hidden solution to the $\mu / B_{\mu}$ problem in gauge mediation, Phys. Rev. D 77 (2008) 095008 [arXiv:0708. 3593] [SPIRES].

[45] H. Murayama, Y. Nomura and D. Poland, More visible effects of the hidden sector, Phys. Rev. D 77 (2008) 015005 [arXiv:0709.0775] [SPIRES].

[46] G. Perez, T.S. Roy and M. Schmaltz, Phenomenology of SUSY with scalar sequestering, arXiv:0811.3206 [SPIRES].

[47] B.C. Allanach, G. Hiller, D.R.T. Jones and P. Slavich, Flavour violation in anomaly mediated supersymmetry breaking, JHEP 04 (2009) 088 [arXiv: 0902 .4880] [SPIRES].

[48] F. Gabbiani, E. Gabrielli, A. Masiero and L. Silvestrini, A complete analysis of FCNC and $C P$ constraints in general SUSY extensions of the standard model,

Nucl. Phys. B 477 (1996) 321 [hep-ph/9604387] [SPIRES].

[49] J.L. Feng, B.T. Smith and F. Takayama, Goldilocks supersymmetry: simultaneous solution to dark matter and flavor problems of supersymmetry, Phys. Rev. Lett. 100 (2008) 021302 [arXiv: 0709.0297] [SPIRES].

[50] G.F. Giudice and A. Masiero, A natural solution to the $\mu$ problem in supergravity theories, Phys. Lett. B 206 (1988) 480 [SPIRES].

[51] W. Buchmüller, K. Hamaguchi and J. Kersten, The gravitino in gaugino mediation, Phys. Lett. B 632 (2006) 366 [hep-ph/0506105] [SPIRES].

[52] B. Holdom, Two U(1)'s and $\epsilon$ charge shifts, Phys. Lett. B 166 (1986) 196 [SPIRES].

[53] K.S. Babu, C.F. Kolda and J. March-Russell, Leptophobic U(1)s and the $R_{b}-R_{c}$ crisis, Phys. Rev. D 54 (1996) 4635 [hep-ph/9603212] [SPIRES].

[54] K.R. Dienes, C.F. Kolda and J. March-Russell, Kinetic mixing and the supersymmetric gauge hierarchy, Nucl. Phys. B 492 (1997) 104 [hep-ph/9610479] [SPIRES];

K.S. Babu, C.F. Kolda and J. March-Russell, Implications of generalized $Z Z^{\prime}$ mixing, Phys. Rev. D 57 (1998) 6788 [hep-ph/9710441] [SPIRES]. 
[55] S.A. Abel, M.D. Goodsell, J. Jaeckel, V.V. Khoze and A. Ringwald, Kinetic mixing of the photon with hidden $\mathrm{U}(1) s$ in string phenomenology, JHEP 07 (2008) 124 [arXiv:0803.1449] [SPIRES].

[56] A. Ibarra, A. Ringwald and C. Weniger, Hidden gauginos of an unbroken $\mathrm{U}(1)$ : cosmological constraints and phenomenological prospects, JCAP 01 (2009) 003 [arXiv:0809.3196] [SPIRES].

[57] P. Fayet and J. Iliopoulos, Spontaneously broken supergauge symmetries and Goldstone spinors, Phys. Lett. B 51 (1974) 461 [SPIRES].

[58] D. Suematsu, SUSY breaking based on abelian gaugino kinetic term mixings, JHEP 11 (2006) 029 [hep-ph/0606125] [SPIRES].

[59] P. Batra and E. Ponton, Supersymmetric electroweak symmetry breaking, Phys. Rev. D 79 (2009) 035001 [arXiv:0809.3453] [SPIRES].

[60] S. Dimopoulos and G.F. Giudice, Multi-messenger theories of gauge-mediated supersymmetry breaking, Phys. Lett. B 393 (1997) 72 [hep-ph/9609344] [SPIRES].

[61] G.R. Dvali, G.F. Giudice and A. Pomarol, The $\mu$-problem in theories with gauge-mediated supersymmetry breaking, Nucl. Phys. B 478 (1996) 31 [hep-ph/9603238] [SPIRES].

[62] G.F. Giudice and R. Rattazzi, Extracting supersymmetry-breaking effects from wave-function renormalization, Nucl. Phys. B 511 (1998) 25 [hep-ph/9706540] [SPIRES].

[63] N. Arkani-Hamed, G.F. Giudice, M.A. Luty and R. Rattazzi, Supersymmetry-breaking loops from analytic continuation into superspace, Phys. Rev. D 58 (1998) 115005 [hep-ph/9803290] [SPIRES].

[64] P. Meade, N. Seiberg and D. Shih, General gauge mediation, arXiv:0801.3278 [SPIRES].

[65] J.L. Evans, D.E. Morrissey and J.D. Wells, Higgs boson exempt no-scale supersymmetry and its collider and cosmology implications, Phys. Rev. D 75 (2007) 055017 [hep-ph/0611185] [SPIRES].

[66] S.P. Martin and M.T. Vaughn, Two loop renormalization group equations for soft supersymmetry breaking couplings, Phys. Rev. D 50 (1994) 2282 [Erratum ibid. D 78 (2008) 039903] [hep-ph/9311340] [SPIRES].

[67] E. Poppitz and S.P. Trivedi, Some remarks on gauge-mediated supersymmetry breaking, Phys. Lett. B 401 (1997) 38 [hep-ph/9703246] [SPIRES].

[68] M. Ibe and R. Kitano, Sweet spot supersymmetry, JHEP 08 (2007) 016 [arXiv:0705.3686] [SPIRES].

[69] C. Beasley, J.J. Heckman and C. Vafa, GUTs and exceptional branes in F-theory - I, JHEP 01 (2009) 058 [arXiv:0802.3391] [SPIRES].

[70] C. Beasley, J.J. Heckman and C. Vafa, GUTs and exceptional branes in F-theory - II: experimental predictions, JHEP 01 (2009) 059 [arXiv:0806.0102] [SPIRES].

[71] J.J. Heckman and C. Vafa, F-theory, GUTs and the weak scale, arXiv:0809.1098 [SPIRES].

[72] J. Marsano, N. Saulina and S. Schäfer-Nameki, Gauge mediation in F-theory GUT models, arXiv:0808.1571 [SPIRES].

[73] K. Griest, M. Kamionkowski and M.S. Turner, Supersymmetric dark matter above the $W$ mass, Phys. Rev. D 41 (1990) 3565 [SPIRES]. 
[74] M. Kawasaki, K. Kohri and T. Moroi, Big-Bang nucleosynthesis and hadronic decay of long-lived massive particles, Phys. Rev. D 71 (2005) 083502 [astro-ph/0408426] [SPIRES].

[75] P. Kalyniak, R. Bates and J.N. Ng, Two photon decays of scalar and pseudoscalar bosons in supersymmetry, Phys. Rev. D 33 (1986) 755 [SPIRES];

R. Bates, J.N. Ng and P. Kalyniak, Two photon decay widths of Higgs bosons in minimal broken supersymmetry, Phys. Rev. D 34 (1986) 172 [SPIRES].

[76] J.F. Gunion, G. Gamberini and S.F. Novaes, Can the Higgs bosons of the minimal supersymmetric model be detected at a hadron collider via two photon decays?, Phys. Rev. D 38 (1988) 3481 [SPIRES].

[77] CDMS collaboration, Z. Ahmed et al., Search for Weakly Interacting Massive Particles with the first five-tower data from the Cryogenic Dark Matter Search at the Soudan Underground Laboratory, Phys. Rev. Lett. 102 (2009) 011301 [arXiv:0802.3530] [SPIRES].

[78] XENON collaboration, J. Angle et al., First results from the XENON10 dark matter experiment at the Gran Sasso National Laboratory, Phys. Rev. Lett. 100 (2008) 021303 [arXiv:0706.0039] [SPIRES].

[79] S. Andreas, T. Hambye and M.H.G. Tytgat, WIMP dark matter, Higgs exchange and DAMA, JCAP 10 (2008) 034 [arXiv:0808.0255] [SPIRES].

[80] Y.G. Kim and S. Shin, Singlet fermionic dark matter explains DAMA signal, arXiv: 0901.2609 [SPIRES].

[81] J. March-Russell, C. McCabe and M. McCullough, Inelastic dark matter, non-standard halos and the DAMA/LIBRA results, JHEP 05 (2009) 071 [arXiv:0812.1931] [SPIRES].

[82] P. Ullio, M. Kamionkowski and P. Vogel, Spin dependent WIMPs in DAMA?, JHEP 07 (2001) 044 [hep-ph/0010036] [SPIRES].

[83] C. Savage, P. Gondolo and K. Freese, Can WIMP spin dependent couplings explain DAMA data, in light of null results from other experiments?, Phys. Rev. D 70 (2004) 123513 [astro-ph/0408346] [SPIRES].

[84] D. Hooper, F. Petriello, K.M. Zurek and M. Kamionkowski, The new DAMA dark-matter window and energetic-neutrino searches, Phys. Rev. D 79 (2009) 015010 [arXiv:0808.2464] [SPIRES].

[85] J.L. Feng, J. Kumar, J. Learned and L.E. Strigari, Testing the dark matter interpretation of the DAMA/LIBRA result with Super-Kamiokande, arXiv:0808.4151 [SPIRES].

[86] G. Jungman, M. Kamionkowski and K. Griest, Supersymmetric dark matter, Phys. Rept. 267 (1996) 195 [hep-ph/9506380] [SPIRES].

[87] D.S.M. Alves, S.R. Behbahani, P. Schuster and J.G. Wacker, Composite inelastic dark matter, arXiv:0903.3945 [SPIRES].

[88] D.P. Finkbeiner, T.R. Slatyer, N. Weiner and I. Yavin, PAMELA, DAMA, INTEGRAL and signatures of metastable excited WIMPs, arXiv:0903.1037 [SPIRES].

[89] B. Batell, M. Pospelov and A. Ritz, Direct detection of multi-component secluded WIMPs, arXiv: 0903.3396 [SPIRES].

[90] M. Cirelli, N. Fornengo and A. Strumia, Minimal dark matter, Nucl. Phys. B 753 (2006) 178 [hep-ph/0512090] [SPIRES].

[91] G. Bertone, M. Cirelli, A. Strumia and M. Taoso, Gamma-ray and radio tests of the $e^{+} e^{-}$ excess from DM annihilations, JCAP 03 (2009) 009 [arXiv:0811.3744] [SPIRES]. 
[92] L. Bergstrom, G. Bertone, T. Bringmann, J. Edsjo and M. Taoso, Gamma-ray and radio constraints of high positron rate dark matter models annihilating into new light particles, arXiv:0812. 3895 [SPIRES].

[93] P. Meade, M. Papucci and T. Volansky, Dark matter sees the light, arXiv:0901.2925 [SPIRES].

[94] J. Mardon, Y. Nomura, D. Stolarski and J. Thaler, Dark matter signals from cascade annihilations, JCAP 05 (2009) 016 [arXiv:0901.2926] [SPIRES].

[95] M.J. Strassler, Possible effects of a hidden valley on supersymmetric phenomenology, hep-ph/0607160 [SPIRES].

[96] M.J. Strassler and K.M. Zurek, Echoes of a hidden valley at hadron colliders, Phys. Lett. B 651 (2007) 374 [hep-ph/0604261] [SPIRES]; Discovering the Higgs through highly-displaced vertices, Phys. Lett. B 661 (2008) 263 [hep-ph/0605193] [SPIRES].

[97] Y. Bai and Z. Han, Measuring the dark force at the LHC, arXiv:0902.0006 [SPIRES].

[98] T. Han, Z. Si, K.M. Zurek and M.J. Strassler, Phenomenology of hidden valleys at hadron colliders, JHEP 07 (2008) 008 [arXiv:0712.2041] [SPIRES].

[99] B. Batell, M. Pospelov and A. Ritz, Probing a secluded U(1) at B-factories, arXiv: 0903.0363 [SPIRES].

[100] R. Essig, P. Schuster and N. Toro, Probing dark forces and light hidden sectors at low-energy $e^{+} e^{-}$colliders, arXiv:0903.3941 [SPIRES].

[101] B. McElrath, Invisible quarkonium decays as a sensitive probe of dark matter, Phys. Rev. D 72 (2005) 103508 [hep-ph/0506151] [SPIRES].

[102] N. Borodatchenkova, D. Choudhury and M. Drees, Probing MeV dark matter at low-energy $e^{+} e^{-}$colliders, Phys. Rev. Lett. 96 (2006) 141802 [hep-ph/0510147] [SPIRES].

[103] M. Reece and L.-T. Wang, Searching for the light dark gauge boson in GeV-scale experiments, arXiv:0904.1743 [SPIRES].

[104] Belle collaboration, O. Tajima et al., Search for invisible decay of the $\Upsilon(1 S)$, Phys. Rev. Lett. 98 (2007) 132001 [hep-ex/0611041] [SPIRES].

[105] CLEO collaboration, P. Rubin et al., Search for invisible decays of the $\Upsilon(1 S)$ resonance, Phys. Rev. D 75 (2007) 031104 [hep-ex/0612051] [SPIRES].

[106] P. Fayet, U-boson production in $e^{+} e^{-}$annihilations, $\psi$ and $\Upsilon$ decays and light dark matter, Phys. Rev. D 75 (2007) 115017 [hep-ph/0702176] [SPIRES].

[107] S.P. Martin, Generalized messengers of supersymmetry breaking and the sparticle mass spectrum, Phys. Rev. D 55 (1997) 3177 [hep-ph/9608224] [SPIRES].

[108] I. Jack and D.R.T. Jones, Renormalisation of the Fayet-Iliopoulos D-term, Phys. Lett. B 473 (2000) 102 [hep-ph/9911491] [SPIRES]; Fayet-Iliopoulos D-terms and anomaly mediated supersymmetry breaking, Phys. Lett. B 482 (2000) 167 [hep-ph/0003081] [SPIRES];

I. Jack, D.R.T. Jones and S. Parsons, The Fayet-Iliopoulos D-term and its renormalisation in softly-broken supersymmetric theories, Phys. Rev. D 62 (2000) 125022 [hep-ph/0007291] [SPIRES]. 